\title{
FINITE VOLUME METHODS ON SPHERES AND SPHERICAL CENTROIDAL VORONOI MESHES*
}

\author{
QIANG DU ${ }^{\dagger}$ AND LILI JU
}

\begin{abstract}
We study in this paper a finite volume approximation of linear convection diffusion equations defined on a sphere using the spherical Voronoi meshes, in particular, the spherical centroidal Voronoi meshes. The high quality of spherical centroidal Voronoi meshes is illustrated through both theoretical analysis and computational experiments. In particular, we show that the $L^{2}$ error of the approximate solution is of quadratic order when the underlying Voronoi mesh is given by a spherical centroidal Voronoi mesh. We also demonstrates numerically the high accuracy and the superconvergence of the approximate solutions.
\end{abstract}

Key words. Finite volume method, spherical Voronoi tessellations, spherical centroidal Voronoi tessellations, error estimates, convection-diffusion equations.

AMS subject classifications. 65N15, 65N50, 65D17

1. Introduction. The numerical solution of partial differential equations defined on spheres is a very active research subject in the scientific community. The subject is related to a number of important applications such as weather forecast and climate modeling. For example, the numerical solution of linear convection-diffusion equations and nonlinear shallow water equations in the spherical geometry can be used to test numerical algorithms for more complex atmospheric circulation models. Though these models were often solved with spectral methods or traditional finite difference methods in spherical coordinates, methods that use quasi-uniform tessellations of the sphere are gradually gaining popularity as the grid-based methods offer great potential when combined with massive parallelism and local adaptivity.

To get efficient and accurate numerical solutions of PDEs, it is well known that grid quality plays an important role and high quality grid generation is often a significant part of the overall solution process. Recently, many studies have been made on the development of finite element and finite volume approximations to PDEs defined on spheres using various spherical grids, such as grids based on Bucky balls [15], icosahedral grids [3, 30], skipped grid [21], grids obtained from a gnomonic (cubed sphere) mapping [20], etc. In standard Euclidean geometry, the so-called Voronoi-Delaunay grids have always been very popular grids used in both finite element and finite volume methods. Grids generated from the spherical Voronoi tessellations and their variations have also been studied, see for example [14, 31].

In [7] and [8], we proposed a high quality spherical grid based on the Spherical Centroidal Voronoi Tessellation (SCVT) which can be used for both data assimilation purposes and for the numerical solution of PDEs on spheres. A finite volume approximation to a second order linear elliptic equation using the spherical Voronoi meshes was studied in [8], and a first order error estimate for the discrete $H^{1}$ norm was obtained under some grid regularity assumptions. Preliminary numerical experiments was also provided there to demonstrate the good performance when the finite volume scheme was implemented with the Spherical Centroidal Voronoi Meshes (SCVM) that include both the SCVT and its dual (Delaunay) triangular grid. A very recent study made in [29] on both the global and the local uniformity of spherical grids indicated that the SCVT grid with a uniform density measures better than many other variations. Moreover, after examining the local truncation errors as well as the

\footnotetext{
* This work is supported in part by NSF-DMS 0196522 and NSF-ITR 0205232.

†Department of Mathematics, Pennsylvania State University, University Park, PA 16802. (qdu@math.psu.edu).

$\ddagger$ Institute for Mathematics and its Applications, University of Minnesota, Minneapolis, MN 55455. (ju@ima.umn.edu).
} 
solutions errors for a model Poisson equation on the sphere, it was concluded that the SCVT based grid tends to produces the smallest errors among all the grids under consideration and such a grid would naturally remains as a safe choice in practice.

The SCVT can also be defined with a nonuniform density function and thus makes it suitable for adaptive computation. In light of the optimization properties they enjoy [7], grids generated by the SCVM, in some sense, may be viewed as optimal grids: they offer both excellent local grid regularity and global mesh conformity as well as flexible mesh adaptivity. In this paper, we make further attempts to substantiate the optimality of SCVM both theoretically and computationally. Our main results include a carefully designed finite volume scheme for a general second order convection-diffusion equation defined on a sphere. When implemented with the SCVM, we present for such a discrete scheme a rigorous quadratic or$\operatorname{der} L^{2}$ error estimate whose proof relies critically on the geometric properties of the SCVT. We further demonstrate through experiments the superconvergent properties of the numerical solutions and their gradients solved using our modified finite volume scheme and the SCVT based grid. All these findings provide compelling reasons for regarding the SCVT's with the uniform density as arguably the best alternative for near uniform partitions of the sphere and the SCVT based grids the optimal triangular grids to use for the numerical solution of many partial differential equations defined on spheres.

We point out that the conclusions given in this paper can be readily adapted to problems defined on the two dimensional Euclidean plane. The analysis for the spherical case is somewhat more involved than the planar case since we must deal with the differences between spherical triangles and planar triangles.

The paper is organized as follows: we first introduce some notation and assumptions used in the paper, then in section 2, we briefly recall the basic theory of the spherical centroidal Voronoi meshes. The finite volume scheme for linear convection-diffusion equations on the sphere given in [8] is discussed in section 3. With a suitable modification to the finite volume scheme, a rigorous $L^{2}$ error estimate is given in section 4 for spherical centroidal Voronoi meshes. In section 5, a superconvergent gradient recovery scheme is provided and in section 6 , we present some numerical experiments. Some concluding remarks are given in section 7 .

We now introduce some basic notation used in the paper. Let $\mathbb{S}^{2}$ denote the (surface of the) sphere having radius $r>0$, i.e.,

$$
\mathbb{S}^{2}=\left\{\mathbf{x}=\left(x_{1}, x_{2}, x_{3}\right) \in \mathbb{R}^{3} \quad \mid \quad\|\mathbf{x}\|=r\right\},
$$

where $\|\cdot\|$ denotes the Euclidean norm. Throughout the paper, the term sphere denotes the surface of a ball in $\mathbb{R}^{3}$. Let $\nabla_{s}$ denote the tangential gradient operator $[10,13]$ on $\mathbb{S}^{2}$ defined by

$$
\nabla_{s} u(\mathbf{x})=\left(\nabla_{s, 1}, \nabla_{s, 2}, \nabla_{s, 3}\right) u(\mathbf{x})=\nabla u(\mathbf{x})-\left(\nabla u(\mathbf{x}) \cdot \overrightarrow{\mathbf{n}}_{\mathbb{S}^{2}, \mathbf{x}}\right) \overrightarrow{\mathbf{n}}_{\mathbb{S}^{2}, \mathbf{x}},
$$

where $\nabla=\left(D_{1}, D_{2}, D_{3}\right)$ denotes the general gradient operator in $\mathbb{R}^{3}$ and $\overrightarrow{\mathbf{n}}_{\mathbb{S}^{2}, \mathbf{x}}$ is the unit outer normal vector to $\mathbb{S}^{2}$ at $\mathbf{x}$. We consider the second order elliptic equation on the sphere given by

$$
\nabla_{s} \cdot\left(-a(\mathbf{x}) \nabla_{s} u(\mathbf{x})+\overrightarrow{\mathbf{v}}(\mathbf{x}) u(\mathbf{x})\right)+b(\mathbf{x}) u(\mathbf{x})=f(\mathbf{x}) \quad \text { for } \mathbf{x} \in \mathbb{S}^{2} .
$$

Note that, since $\mathbb{S}^{2}$ has no boundary, there is no boundary condition imposed.

We use the standard notation for Sobolev spaces on $\mathbb{S}^{2}$ (viewed as a compact, twodimensional Riemannian manifold) [13]:

$$
L^{p}\left(\mathbb{S}^{2}\right)=\left\{\left.u(\mathbf{x}) \quad\left|\quad \int_{\mathbb{S}^{2}}\right| u(\mathbf{x})\right|^{p} d s(\mathbf{x})<\infty\right\},
$$




$$
W^{m, p}\left(\mathbb{S}^{2}\right)=\left\{u \in L^{2}\left(\mathbb{S}^{2}\right) \quad \mid \quad \nabla_{s}^{\alpha} u \in L^{p}\left(\mathbb{S}^{2}\right) \quad \text { for } 0 \leq|\alpha| \leq m\right\},
$$

where $\alpha=\left(\alpha_{1}, \alpha_{2}, \alpha_{3}\right), \nabla_{s}^{\alpha}=\nabla_{s, 1}^{\alpha_{1}} \nabla_{s, 2}^{\alpha_{2}} \nabla_{s, 3}^{\alpha_{3}}$, and $|\alpha|=\alpha_{1}+\alpha_{2}+\alpha_{3}$. Also,

$$
\|u\|_{W^{m, p}\left(\mathbb{S}^{2}\right)}= \begin{cases}\left(\sum_{0 \leq|\alpha| \leq m}\left\|\nabla_{s}^{\alpha} u\right\|_{L^{p}\left(\mathbb{S}^{2}\right)}^{p}\right)^{1 / p} & \text { for } 1 \leq p<\infty \\ \max _{0 \leq|\alpha| \leq m}\left\|\nabla_{s}^{\alpha} u\right\|_{L^{\infty}\left(\mathbb{S}^{2}\right)} & \text { for } p=\infty .\end{cases}
$$

For the case $p=2$, we let $H^{m}\left(\mathbb{S}^{2}\right)=W^{m, 2}\left(\mathbb{S}^{2}\right)$.

Let the data in (1.1) satisfy the following set of assumptions.

Assumption 1. $f \in L^{2}\left(\mathbb{S}^{2}\right), a \in C^{1}\left(\mathbb{S}^{2}\right), b \in L^{\infty}\left(\mathbb{S}^{2}\right)$, and $\overrightarrow{\mathbf{v}} \in C^{1}\left(\mathbb{S}^{2}, \mathbb{R}^{3}\right)$ such that $a(\mathbf{x}) \geq \alpha_{1}>0, b(\mathbf{x}) \geq 0$, and $\nabla_{s} \cdot \overrightarrow{\mathbf{v}}(\mathbf{x})+b(\mathbf{x}) \geq \alpha_{2}>0$, a.e..

For any $u, v \in H^{1}\left(\mathbb{S}^{2}\right)$, define the bilinear functional $\mathcal{A}$ such that

$$
\begin{aligned}
\mathcal{A}(u, v)= & \int_{\mathbb{S}^{2}} a(\mathbf{x})\left(\nabla_{s} u(\mathbf{x}) \cdot \nabla_{s} v(\mathbf{x})\right)+u(\mathbf{x})\left(\overrightarrow{\mathbf{v}}(\mathbf{x}) \cdot \nabla_{s} v(\mathbf{x})\right) d s(\mathbf{x}) \\
& +\int_{\mathbb{S}^{2}} b(\mathbf{x}) u(\mathbf{x}) v(\mathbf{x}) d s(\mathbf{x})
\end{aligned}
$$

then we have (for some constant $C>0$ )

$$
\mathcal{A}(u, v) \leq C\|u\|_{H^{1}\left(S^{2}\right)} \|_{H^{1}\left(S^{2}\right)} .
$$

Since $\mathbb{S}^{2}$ is compact, if Assumption 1 holds the problem (1.1) has a unique weak solution $u \in H^{2}\left(\mathbb{S}^{2}\right)$ such that

$$
\mathcal{A}(u, v)=(f, v), \quad \forall v \in H^{1}\left(\mathbb{S}^{2}\right)
$$

where

$$
(f, v)=\int_{\mathbb{S}^{2}} f(\mathbf{x}) v(\mathbf{x}) d s(\mathbf{x})
$$

is the standard $L^{2}$ inner product, and consequently $u$ satisfies (for some constant $C>0$ ) the estimate

$$
\|u\|_{H^{2}\left(\mathbb{S}^{2}\right)} \leq C\|f\|_{L^{2}\left(\mathbb{S}^{2}\right)} .
$$

2. Spherical centroidal Voronoi meshes. Let $d(\mathbf{x}, \mathbf{y})$ denote the geodesic distance between $\mathbf{x}$ and $\mathbf{y}$ on $\mathbb{S}^{2}$, i.e., for $\mathbf{x}, \mathbf{y} \in \mathbb{S}^{2}$,

$$
d(\mathbf{x}, \mathbf{y})=r\left[\arccos \left(\frac{\mathbf{x} \cdot \mathbf{y}}{r^{2}}\right)\right] .
$$

Given a set of distinct points $\left\{\mathbf{x}_{i}\right\}_{i=1}^{n} \subset \mathbb{S}^{2}$, the corresponding spherical Voronoi regions $\left\{V_{i}\right\}_{i=1}^{n}$ are defined by

$$
V_{i}=\left\{\mathbf{y} \in \mathbb{S}^{2} \mid d\left(\mathbf{x}_{i}, \mathbf{y}\right)<d\left(\mathbf{x}_{j}, \mathbf{y}\right) \text { for } j=1, \cdots, n \text { and } j \neq i\right\}, \quad 1 \leq i \leq n .
$$

$\left\{V_{i}\right\}_{i=1}^{n}$ forms a Voronoi tessellation or Voronoi diagrams of $\mathbb{S}^{2}$ associated with the set of generators $\left\{\mathbf{x}_{i}\right\}_{i=1}^{n}$. Each Voronoi cell $V_{i}$ is an open convex spherical polygon on $\mathbb{S}^{2}$ with geodesic arcs making up its boundary. It is also well-known that the dual tessellation (in a graph-theoretical sense) to a Voronoi tessellation of $\mathbb{S}^{2}$ consists of spherical triangles which form the Delaunay triangulation. 
Given a density function $\rho$ defined on $\mathbb{S}^{2}$, for any spherical region $V \subset \mathbb{S}^{2}$, the constrained mass centroid $\mathbf{x}^{c}$ of $V$ on $\mathbb{S}^{2}$ is given by the solution of the following problem:

$$
\min _{\mathbf{x} \in V} F(\mathbf{x}), \quad \text { where } \quad F(\mathbf{x})=\int_{V} \rho(\mathbf{y})\|\mathbf{y}-\mathbf{x}\|^{2} d s(\mathbf{y}) .
$$

As in $[7,8]$, a Voronoi tessellation of $\mathbb{S}^{2}$ is called a constrained centroidal Voronoi tessellation (CCVT) of $\mathbb{S}^{2}$ or specifically Spherical Centroidal Voronoi Tessellation (SCVT) if and only if the points $\left\{\mathbf{x}_{i}\right\}_{i=1}^{m}$ which serve as the generators of the associated spherical Voronoi tessellation $\left\{V_{i}\right\}_{i=1}^{k}$ are also the constrained mass centroids of those Voronoi regions.

Given any set of points $\left\{\widetilde{\mathbf{x}}_{i}\right\}_{i=1}^{n}$ on $\mathbb{S}^{2}$ and any spherical tessellation $\left\{\widetilde{V}_{i}\right\}_{i=1}^{n}$ of $\mathbb{S}^{2}$, we define the corresponding energy by

$$
\mathcal{K}\left(\left\{\widetilde{\mathbf{x}}_{i}, \widetilde{V}_{i}\right\}_{i=1}^{n}\right)=\sum_{i=1}^{n} \int_{\widetilde{V}_{i}} \rho(\mathbf{x})\left\|\mathbf{x}-\widetilde{\mathbf{x}}_{i}\right\|^{2} d s(\mathbf{x}) .
$$

It can be shown [7] that $\mathcal{K}(\cdot)$ is minimized only if $\left\{\widetilde{\mathbf{x}}_{i}, \widetilde{V}_{i}\right\}_{i=1}^{n}$ are a spherical centroidal Voronoi tessellation. Consequently, spherical centroidal Voronoi meshes have many good geometric properties, see $[7,8]$. If the density function $\rho(\mathbf{x})$ is a constant on $\mathbb{S}^{2}$, then the SCVT generators will be uniformly distributed. Alternatively, a non-constant density function provides systematically a non-uniform distribution of points while the accumulation of SCVT generators still remains locally regular.

Constructing a constrained mass centroid from (2.1) may be cumbersome. In [7], it has been shown that one can compute first the centroid in $\mathbb{R}^{3}$ by

$$
\mathbf{x}_{i}^{*}=\frac{\int_{V_{i}} \mathbf{x} \rho(\mathbf{x}) d s(\mathbf{x})}{\int_{V_{i}} \rho(\mathbf{x}) d s(\mathbf{x})}, \quad \text { for } i=1, \ldots, n,
$$

then compute $\mathbf{x}_{i}^{c}$ using the fact that it is the projection of $\mathbf{x}_{i}^{*}$ onto $\mathbb{S}^{2}$ along the normal direction at $\mathbf{x}_{i}^{c}$. Both deterministic and probabilistic algorithms have been provided in $[7,8]$ to construct a SCVT. The deterministic version requires an explicit geometric construction of general spherical Voronoi tessellations which was done by the algorithms given in [28]. One may also extend the work in [18] to parallelize some versions of the probabilistic algorithms.

Fig.2.1 shows some examples of SCVT's associated with a constant density. More examples, including SCVT's with nonuniform densities, can be found in [8].

3. A finite volume method on spherical Voronoi tessellations. Given a spherical Voronoi mesh $\mathcal{W}=\left\{\mathbf{x}_{i}, V_{i}\right\}_{i=1}^{n}$, following the discussion in [8], we refer to a pair of generators $\mathbf{x}_{i}$ and $\mathbf{x}_{j}$ as neighbors if and only if $\Gamma_{i, j}=\bar{V}_{i} \cap \bar{V}_{j} \neq \emptyset$. Note that, for Voronoi meshes, $\Gamma_{i, j}$ can only be a point or a geodesic arc on the sphere. Let

$$
m(A)=\left\{\begin{array}{l}
\text { the area of } A \text { if it is a nonempty subdomain of } \mathbb{S}^{2} \\
\text { the length of } A \text { if it is a geodesic } \operatorname{arc~on} \mathbb{S}^{2}
\end{array}\right.
$$

Then, for each $\mathbf{x}_{i}$, we denote by $\chi_{i}$ the set of the indices of its neighbors $\mathbf{x}_{j}$ 's such that $m\left(\Gamma_{i, j}\right)>0$, i.e., of those neighbors for which $\Gamma_{i, j}$ is a geodesic arc. We also denote by $\overline{\mathbf{x}_{i} \mathbf{x}_{j}}$ the vector from $\mathbf{x}_{i}$ to $\mathbf{x}_{j}$, by $\widetilde{\mathbf{x}_{i} \mathbf{x}_{j}}$ the geodesic arc joining $\mathbf{x}_{i}$ and $\mathbf{x}_{j}$, and by $\mathbf{x}_{i j}$ the midpoint of the geodesic arc $\widetilde{\mathbf{x}_{i} \mathbf{x}_{j}}$. From the construction of spherical Voronoi tessellations, 

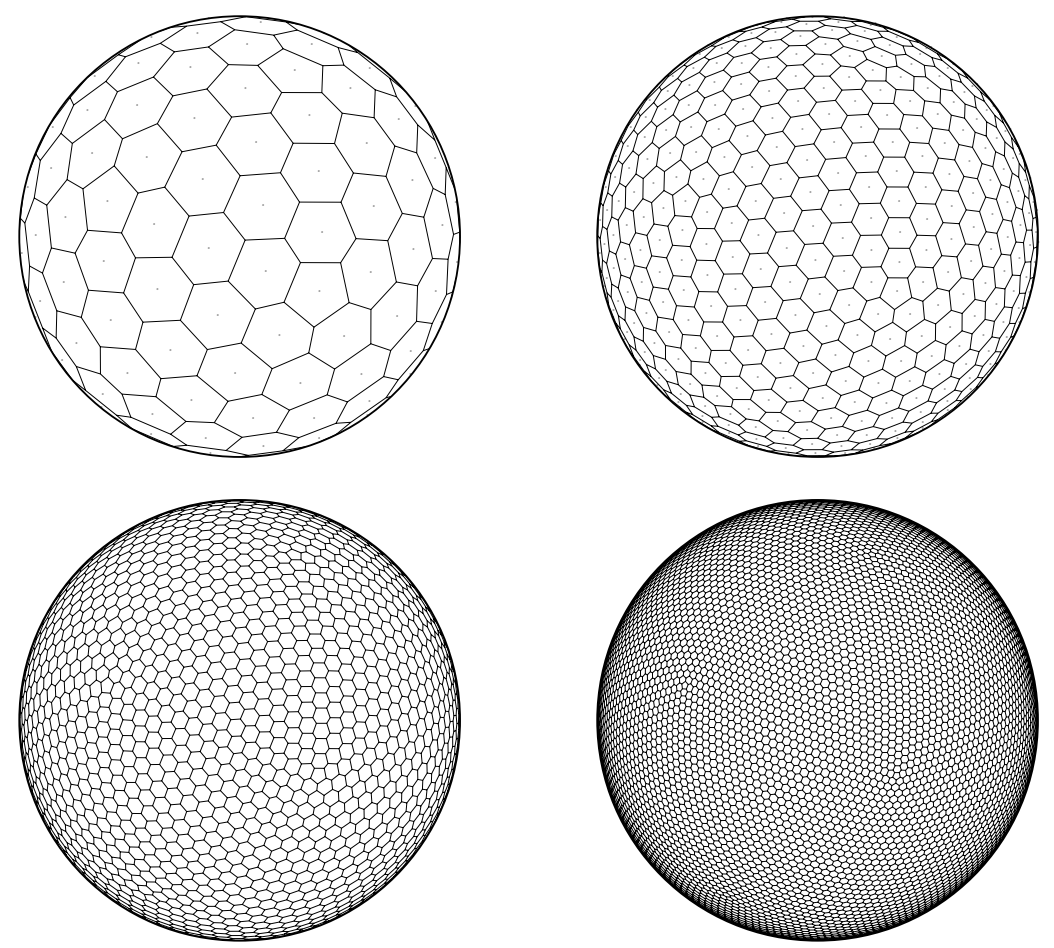

FIG. 2.1. Spherical centroidal Voronoi tessellations of a sphere for a constant density function with 162 (lefttop), 642 (right-top), 2562 (left-bottom) and 10242 (right-bottom) generators.

it is known that $\widetilde{\mathbf{x}_{i} \mathbf{x}_{j}}$ is perpendicular to $\Gamma_{i j}$ and the plane determined by $\Gamma_{i, j}$ and the origin bisects $\widetilde{\mathbf{x}_{i} \mathbf{x}_{j}}$ at its midpoint $\mathbf{x}_{i j}$ [28], see Fig. 3.1. Thus,

$$
\left\|\mathbf{x}_{i}-\mathbf{x}\right\|=\left\|\mathbf{x}_{j}-\mathbf{x}\right\| \quad \text { and } \quad \overrightarrow{\mathbf{n}}_{\mathbf{x}, V_{k}} \| \overline{\mathbf{x}_{i} \mathbf{x}_{j}} \quad \text { for } \quad \mathbf{x} \in \Gamma_{i j}, k=i, j,
$$

where the outer unit normal vector to the boundary of $V_{i}$, denoted by $\overrightarrow{\mathbf{n}}_{\mathbf{x}, V_{i}}$, is taken to lie in the tangent plane of $\mathbb{S}^{2}$ at $\mathbf{x}$.

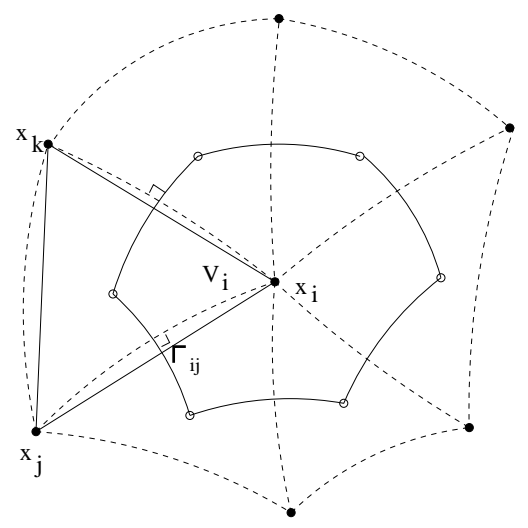

FIG. 3.1. A spherical Voronoi region and its dual triangles.

Meshes of the type $\mathcal{W}=\left\{\mathbf{x}_{i}, V_{i}\right\}_{i=1}^{n}$ are used as control (or finite) volumes for the discretization method discussed below. 
3.1. A finite volume discretization scheme. Denote by $\mathcal{V}_{\mathcal{W}}$ the space of all piecewise constant grid functions associated with a spherical Voronoi mesh $\mathcal{W}=\left\{\mathbf{x}_{i}, V_{i}\right\}_{i=1}^{n}$ of $\mathbb{S}^{2}$, i.e.,

$$
\mathcal{V}_{\mathcal{W}}=\left\{u \mid u(\mathbf{x}) \text { is constant on each cell } V_{i}\right\}
$$

Set $\left\{u_{i}^{h}=u^{h}\left(\mathbf{x}_{i}\right)\right\}_{i=1}^{n}$ and denote by $\mathcal{F}_{i j}$ the approximate diffusion flux defined by

$$
\mathcal{F}_{i j}\left(u^{h}\right)=-m\left(\Gamma_{i j}\right) a_{i j} \frac{u_{j}^{h}-u_{i}^{h}}{\left\|\mathbf{x}_{j}-\mathbf{x}_{i}\right\|} \approx \int_{\Gamma_{i j}}\left(-a(\mathbf{x}) \nabla_{s} u(\mathbf{x})\right) \cdot \overrightarrow{\mathbf{n}}_{\mathbf{x}, V_{i}} d \gamma(\mathbf{x}),
$$

where

$$
m\left(\Gamma_{i j}\right) a_{i j}=\int_{\Gamma_{i j}} a(\mathbf{x}) d \gamma(\mathbf{x})
$$

Based on the Green's formula, a finite volume method for seeking the approximate solution $u^{h} \in \mathcal{V}_{\mathcal{W}}$ of (1.1) was proposed in [8]. It used an up-wind approximate convection flux $\mathcal{V}_{i, j}$ [17] defined by

$$
\mathcal{V}_{i, j}=\beta_{i j}^{+} u_{i}^{h}+\beta_{i j}^{-} u_{j}^{h} \approx \int_{\Gamma_{i j}}(\overrightarrow{\mathbf{v}}(\mathbf{x}) u(\mathbf{x})) \cdot \overrightarrow{\mathbf{n}}_{\mathbf{x}, V_{i}} d \gamma(\mathbf{x}),
$$

where

$$
\beta_{i j}^{+}=\frac{1}{2}\left(\beta_{i j}+\left|\beta_{i j}\right|\right), \quad \beta_{i j}^{-}=\frac{1}{2}\left(\beta_{i j}-\left|\beta_{i j}\right|\right) \quad \text { and } \quad \beta_{i j}=\int_{\Gamma_{i j}} \overrightarrow{\mathbf{v}}(\mathbf{x}) \cdot \overrightarrow{\mathbf{n}}_{\mathbf{x}, V_{i}} d \gamma(\mathbf{x}) .
$$

For all $V_{i}$, let $f_{i}$ and $b_{i}$ denote respectively the mean value of $f$ and $b$ on $V_{i}$, i.e.,

$$
f_{i}=\frac{1}{m\left(V_{i}\right)} \int_{V_{i}} f(\mathbf{x}) d s(\mathbf{x}) \quad \text { and } \quad b_{i}=\frac{1}{m\left(V_{i}\right)} \int_{V_{i}} b(\mathbf{x}) d s(\mathbf{x}) .
$$

Then, the finite volume scheme given by [8] is defined by the following system:

$$
\left(\mathcal{L}^{h} u^{h}\right)_{i}=\frac{1}{m\left(V_{i}\right)} \sum_{j \in \chi_{i}}\left(\mathcal{F}_{i j}+\mathcal{V}_{i j}\right)+b_{i} u_{i}^{h}=f_{i} \quad \text { for } i=1, \ldots, n
$$

REMARK 1. An approximate convection flux which leads to a central difference scheme is defined by

$$
\mathcal{V}_{i, j}=\frac{\beta_{i j}}{2}\left(u_{i}^{h}+u_{j}^{h}\right)
$$

Then, a stability condition such as

$$
P_{i}=\max _{j \in \chi_{i}} \frac{\left|\beta_{i j}\right| \cdot\left\|\mathbf{x}_{i}-\mathbf{x}_{j}\right\|}{2 m\left(\Gamma_{i j}\right) a_{i j}} \leq 1 \quad \text { for } \quad i=1, \cdots, n,
$$

is needed, where $P_{i}$ is called the local Peclet number [17, 25]. 
3.2. Previous results. For given grid functions $u, v \in \mathcal{V}_{\mathcal{W}}$, we define, similar to [25], the following discrete inner products and norms associated with a spherical Voronoi mesh $\mathcal{W}=\left\{\mathbf{x}_{i}, V_{i}\right\}_{i=1}^{n}$ :

$$
\left\{\begin{array}{l}
(u, v)_{\mathcal{W}}=\sum_{i=1}^{n} m\left(V_{i}\right) u\left(\mathbf{x}_{i}\right) v\left(\mathbf{x}_{i}\right), \quad\|u\|_{0, \mathcal{W}}^{2}=(u, u)_{\mathcal{W}} \\
|u|_{1, \mathcal{W}}^{2}=\frac{1}{2} \sum_{i=1}^{m} \sum_{j \in \chi_{i}} m\left(\Gamma_{i j}\right) d\left(\mathbf{x}_{i}, \mathbf{x}_{j}\right)\left(\frac{u\left(\mathbf{x}_{i}\right)-u\left(\mathbf{x}_{j}\right)}{\left\|\mathbf{x}_{i}-\mathbf{x}_{j}\right\|}\right)^{2} \\
\|u\|_{1, \mathcal{W}}^{2}=\|u\|_{0, \mathcal{W}}^{2}+|u|_{1, \mathcal{W}}^{2} .
\end{array}\right.
$$

Norms for general function spaces can also be defined.

We define the mesh quality norm $h$ by

$$
h=\max _{i=1, \ldots, n} h_{i}, \quad \text { where } \quad h_{i}=\max _{\mathbf{y} \in V_{i}} d\left(\mathbf{x}_{i}, \mathbf{y}\right) .
$$

Thus, $h_{i}$ gives the maximum geodesic distance between a particular generator $\mathbf{x}_{i}$ and the points in its associated cell $V_{i}$ and $h$ gives the maximum of all $h_{i}$ 's. The above mesh norm $h$ has been used in [7] in the context of polynomial interpolation on the sphere.

Given a Voronoi mesh $\mathcal{W}=\left\{\mathbf{x}_{i}, V_{i}\right\}_{i=1}^{m}$, we define the mesh regularity norm $\sigma$ by

$$
\sigma=\min _{i=1, \ldots, n} \sigma_{i}, \quad \text { where } \quad \sigma_{i}=\min _{j \in \chi_{i}} \sigma_{i j} \quad \text { and } \quad \sigma_{i j}=\frac{d\left(\mathbf{x}_{i}, \mathbf{x}_{j}\right)}{2 h_{i}} .
$$

$\sigma$ can be used as a measure of the uniformity of a mesh; the larger the value of $\sigma$, the more uniform is the mesh. In addition, the value of $\sigma$ provides us a measure of the mesh regularity, i.e., the local uniformity of a mesh. Again, if a mesh is locally uniform in the sense that the cells in a neighborhood of any cell are nearly congruent to that cell, then the value of $\sigma$ will again be large. We will refer to $\mathcal{W}$ as regular if $\sigma$ is not too small. To get a reasonable theoretical estimate, we will need to require a regularity condition on the mesh. The following result has been proved in [8].

THEOREM 1. Suppose that Assumption 1 is satisfied. Let $\mathcal{F}_{i j}, \mathcal{V}_{i j}$, and $b_{i}$ be defined by (3.3)-(3.5). Then the discrete system (3.6) has a unique solution $u^{h} \in \mathcal{V}_{\mathcal{W}}$. Furthermore, assume that the unique variational solution $u$ of $(1.1)$ belongs to $H^{2}\left(\mathbb{S}^{2}\right)$, then there exists a constant $C>0$ only depending on $\overrightarrow{\mathbf{v}}, b$, and $\sigma$ such that

$$
\left\|e^{h}\right\|_{1, \mathcal{W}} \leq C h\|u\|_{H^{2}\left(\mathbb{S}^{2}\right)}
$$

where $e^{h}(\mathbf{x})=e_{i}^{h}=u\left(\mathbf{x}_{i}\right)-u_{i}^{h}$ for $\mathbf{x} \in V_{i}$.

Note that Theorem 1 holds for general regular spherical Voronoi meshes. For more existing studies on the finite volume methods, especially when applied to solve second order elliptic on the two dimensional plane, we refer to $[2,4,5,9,11,12,16,23,22,24,26,32,33]$.

4. $L^{2}$ error estimate on spherical centroidal Voronoi meshes. An improved error estimate in the $L^{2}$ norm is generally expected for finite element and finite volume approximations of second order elliptic equations. However, in this section, it is shown that the quadratic order error estimate for the scheme under consideration here can only be proved when the grid satisfies certain geometric constraints. In fact, a part of the estimate depends critically on the property that if $\mathcal{W}=\left\{\mathbf{x}_{i}, V_{i}\right\}_{i=1}^{n}$ is a spherical centroidal Voronoi tessellation of $\mathbb{S}^{2}$ corresponding to a density function $\rho$, then,

$$
\int_{V_{i}} \rho(\mathbf{x})\left(\mathbf{x}_{i}^{*}-\mathbf{x}\right) d s(\mathbf{x})=0, \quad \forall i=1,2, \ldots, n
$$


where $\mathbf{x}_{i}^{*}$ is standard mass centroid of $V_{i}$, whose projection (through the standard map $\mathcal{P}$ ) onto the sphere coincides with $\mathbf{x}_{i}$.

Without loss of generality, we set $r=1$, that is, we consider the case of the unit sphere. For the rest of the section, only those schemes based SCVM are analyzed.

If $\mathbf{x}_{i}, \mathbf{x}_{j}$ and $\mathbf{x}_{k}$ are neighbors for each other in $\mathcal{W}$, we denote by $\tilde{T}_{i j k}$ the spherical triangle determined by $\mathbf{x}_{i}, \mathbf{x}_{j}$ and $\mathbf{x}_{k}$, and by $T_{i j k}$ the corresponding planar triangle (see Fig. 3.1). We use the notation

$$
\begin{gathered}
\Sigma=\{i j k \mid i, j, k \text { are neighbors for each other in } \mathcal{W}\}, \\
\tilde{T}_{i j k}=\left\{\mathcal{P}(\mathbf{y})=\mathbf{y} /\|\mathbf{y}\| \quad \mid \mathbf{y} \in T_{i j k}\right\}=\mathcal{P}\left(T_{i j k}\right), \\
\tilde{\mathcal{T}}=\left\{\tilde{T}_{i j k} \mid i j k \in \Sigma\right\}, \quad \text { and } \quad \mathcal{T}=\left\{T_{i j k} \mid i j k \in \Sigma\right\} .
\end{gathered}
$$

It is easy to see that $\tilde{\mathcal{T}}$ is the corresponding spherical Delaunay triangulation of $\mathbb{S}^{2}$ associated with the generators $\left\{\mathbf{x}_{i}\right\}_{i=1}^{n}$. We can also view $\mathcal{P}$ as a one-to-one smooth function that maps $\mathbf{S}^{*}=\cup_{T_{i j k} \in \mathcal{T}} T_{i j k}$ to $\mathbb{S}^{2}=\cup_{\tilde{T}_{i j k} \in \tilde{\mathcal{T}}} \tilde{T}_{i j k}$.

Clearly, $\mathbf{S}^{*} \cap \mathbb{S}^{2}=\left\{\mathbf{x}_{i}\right\}_{i=1}^{n}$. For any $\mathbf{x} \in \mathbb{S}^{2}$ and $\mathbf{x}_{1}, \mathbf{x}_{2} \in \tilde{T}_{i j k}$, we have

$$
\left\{\begin{array}{l}
\left\|\mathbf{x}-\mathcal{P}^{-1}(\mathbf{x})\right\| \leq h^{2} / 2 \\
\left(1-2 h^{2}\right) d\left(\mathbf{x}_{1}, \mathbf{x}_{2}\right) \leq\left\|\mathcal{P}^{-1}\left(\mathbf{x}_{1}\right)-\mathcal{P}^{-1}\left(\mathbf{x}_{2}\right)\right\| \leq\left(1+2 h^{2}\right) d\left(\mathbf{x}_{1}, \mathbf{x}_{2}\right), \\
A\left(T_{i j k}\right) \leq m\left(\tilde{T}_{i j k}\right) \leq\left(1+2 h^{2}\right)^{2} A\left(T_{i j k}\right)
\end{array}\right.
$$

where $A\left(T_{i j k}\right)$ denotes the area of the triangle $T_{i j k}$. Set

$$
\Omega=\left\{\mathbf{x} \mid 1-h^{2}<\|\mathbf{x}\|<1+h^{2}\right\}
$$

we see that $T_{i j k} \subset \Omega$ and $\tilde{T}_{i j k} \subset \Omega$. For any $u \in H^{1}\left(\mathbb{S}^{2}\right)$, define the function $E u$, the extension of $u$ in $\Omega$, by

$$
E u(\mathbf{y})=u\left(\frac{\mathbf{y}}{\|\mathbf{y}\|}\right), \quad \mathbf{y} \in \Omega .
$$

The following results have been established in [8]:

Proposition 1. For any $\mathbf{y} \in \Omega$ and $\mathbf{x}=\mathbf{y} /\|\mathbf{y}\| \in \mathbb{S}^{2}$, and $i, j=1,2,3$,

$$
\left\{\begin{array}{l}
\nabla_{s} u(\mathbf{x})=\nabla E u(\mathbf{x}) \\
\nabla\left(D_{i} E u\right)(\mathbf{x})=\nabla_{s}\left(\nabla_{s, i} u\right)(\mathbf{x})-\left(\nabla_{s, i} u(\mathbf{x})\right) \overrightarrow{\mathbf{n}}_{\mathbb{S}^{2}, \mathbf{x}} \\
\|\mathbf{y}\| \nabla E u(\mathbf{y})=\nabla E u(\mathbf{x}) \\
\|\mathbf{y}\|^{2} D_{i} D_{j} E u(\mathbf{y})=D_{i} D_{j} E u(\mathbf{x})
\end{array}\right.
$$

From Proposition 1, we naturally have

COROLlaRY 1. There exist a constant $C>0$ such that for any $\mathbf{y} \in \Omega, \mathbf{x}=\mathbf{y} /\|\mathbf{y}\|$,

$$
\sum_{i, j=1}^{3}\left|\nabla_{s, i} \nabla_{s, j} u(\mathbf{x})\right|^{2} \leq\|\mathbf{y}\|^{4} \sum_{i, j=1}^{3}\left|D_{i} D_{j} E u(\mathbf{y})\right|^{2} \leq C \sum_{0 \leq|\alpha| \leq 2}\left|\nabla_{s}^{\alpha} u(\mathbf{x})\right|^{2} .
$$

We call $u^{L}$ a piecewise linear function on $\mathbf{S}^{*}$ if and only if

$$
u^{L}(\mathbf{y})=\lambda_{i} u^{L}\left(\mathbf{x}_{i}\right)+\lambda_{j} u^{L}\left(\mathbf{x}_{j}\right)+\lambda_{k} u^{L}\left(\mathbf{x}_{k}\right), \quad \forall \mathbf{y} \in T_{i j k} .
$$


where $\lambda_{i}, \lambda_{j}, \lambda_{k}$ are the barycentric coordinates of $\mathbf{y}$ in the planar triangle $T_{i j k}$. Denote by $\mathcal{U}_{\mathcal{W}}$ the space of all functions $u_{h}$ on $\mathbb{S}^{2}$ such that

$$
u_{h}(\mathbf{x})=u^{L}\left(\mathcal{P}^{-1}(\mathbf{x})\right), \quad \mathbf{x} \in \mathbb{S}^{2} .
$$

where $u^{L}$ is a piecewise linear function on $\mathbf{S}^{*}$ with $\left\{u^{L}\left(\mathbf{x}_{i}\right)=u_{h}\left(\mathbf{x}_{i}\right)\right\}_{i=1}^{n}$, i.e,

$$
E u_{h}(\mathbf{y})=u^{L}(\mathbf{y}), \quad \forall \mathbf{y} \in \mathbf{S}^{*}
$$

Let $\nabla_{*}$ be the tangential surface gradient operator on $\mathbf{S}^{*}$, i.e., for any function $u$ defined on $\mathbf{S}^{*}$,

$$
\nabla_{*} u(\mathbf{y})=\left(\nabla_{*, 1}, \nabla_{*, 2}, \nabla_{*, 3}\right) u(\mathbf{y})=\nabla u(\mathbf{y})-\left(\nabla u(\mathbf{y}) \cdot \overrightarrow{\mathbf{n}}_{T_{i j k}}\right) \overrightarrow{\mathbf{n}}_{T_{i j k}}, \quad \forall \mathbf{y} \in T_{i j k}
$$

where $\overrightarrow{\mathbf{n}}_{T_{i j k}}$ is the unit outer normal vector to $T_{i j k}$. Similarly, we could define the standard Sobolev space on $\mathbf{S}^{*}$. It is easy to get $u_{h} \in H^{1}\left(\mathbb{S}^{2}\right)$ for any $u_{h} \in \mathcal{U}_{\mathcal{W}}$ using (4.3) and the fact that $E u_{h}=u^{L} \in H^{1}\left(\mathbf{S}^{*}\right)$. In addition, as in Proposition 1 and Corollary 1, after some technical derivation, we have, for any $\mathbf{y} \in T_{i j k}$,

$$
\left\{\begin{array}{l}
\left|\nabla_{*} E u(\mathbf{y})\right|^{2} \leq|\nabla E u(\mathbf{y})|^{2} \\
\sum_{i, j=1}^{3}\left|\nabla_{*, i} \nabla_{*, j} E u(\mathbf{y})\right|^{2} \leq \sum_{i, j=1}^{3}\left|D_{i} D_{j} E u(\mathbf{y})\right|^{2} \\
\nabla_{*, i} \nabla_{*, j} E u_{h}(\mathbf{y})=0, \quad i, j=1,2,3 \\
\|\mathbf{y}\| \nabla\left(D_{j} E u_{h}\right)(\mathbf{y})=-\left(\overrightarrow{\mathbf{n}}_{T_{i j k}} \cdot \overrightarrow{\mathbf{n}}_{\mathbb{S}^{2}, P(\mathbf{y})}\right)\left(D_{i} E u_{h}(\mathbf{y})\right) \overrightarrow{\mathbf{n}}_{T_{i j k}} \\
\overrightarrow{\mathbf{n}}_{T_{i j k}} \cdot \overrightarrow{\mathbf{n}}_{\mathbb{S}^{2}, P(\mathbf{y})} \geq 1-2 h^{2} .
\end{array}\right.
$$

From Proposition 1 and (4.5), we obtain,

COROLLARY 2. There exist a constant $C>0$ such that for any $\mathbf{x} \in \tilde{T}_{i j k}$,

$$
\sum_{i, j=1}^{3}\left|\nabla_{s, i} \nabla_{s, j} u_{h}(\mathbf{x})\right|^{2} \leq C h^{2} \sum_{0 \leq|\alpha| \leq 1}\left|\nabla_{s}^{\alpha} u_{h}(\mathbf{x})\right|^{2} .
$$

Let $\tilde{u}=\left.E u\right|_{\mathbf{S}^{*}}$, using (4.5) with Proposition 1 and Corollary 1, we also get that, there exists some constant $C>0$,

$$
\|\tilde{u}\|_{H^{k}\left(T_{i j k}\right)} \leq C\|u\|_{H^{k}\left(\tilde{T}_{i j k}\right)}, \quad k=0,1,2 .
$$

for any $i j k \in \Sigma$. We now give estimates on the interpolants.

PROPOSITION 2. For any $u \in H^{2}\left(\mathbb{S}^{2}\right)$, let $\mathcal{P}_{\mathcal{U}}(u)$ and $\mathcal{P}_{\mathcal{V}}(u)$ be the interpolants of $u$ on $\mathcal{U}_{\mathcal{W}}$ and $\mathcal{V}_{\mathcal{W}}$ respectively, then there exist some constants $C_{1}, C_{2}, C_{3}>0$ such that

$$
\left\{\begin{aligned}
&\left\|u-\mathcal{P}_{\mathcal{U}}(u)\right\|_{L^{2}\left(\mathbb{S}^{2}\right)} \leq C_{1} h^{2}\|u\|_{H^{2}\left(\mathbb{S}^{2}\right)} \\
&\left\|u-\mathcal{P}_{\mathcal{U}}(u)\right\|_{H^{1}\left(\mathbb{S}^{2}\right)} \leq C_{2} h\|u\|_{H^{2}\left(\mathbb{S}^{2}\right)} \\
&\left\|u-\mathcal{P}_{\mathcal{V}}(u)\right\|_{L^{2}\left(\mathbb{S}^{2}\right)} \leq C_{3} h\|u\|_{H^{2}\left(\mathbb{S}^{2}\right)}
\end{aligned}\right.
$$

Proof. The proof requires only standard techniques. Define

$$
u^{L}(\mathbf{y})=\lambda_{i} u\left(\mathbf{x}_{i}\right)+\lambda_{j} u\left(\mathbf{x}_{j}\right)+\lambda_{k} u\left(\mathbf{x}_{k}\right), \quad \forall \mathbf{y} \in T_{i j k} .
$$

then $\mathcal{P}_{\mathcal{U}}(u)(\mathbf{x})=u^{L}\left(\mathcal{P}^{-1}(\mathbf{x})\right)$. Furthermore, using the estimate for the linear interpolation on planar triangles and the relation

$$
u(\mathbf{x})-\mathcal{P}_{\mathcal{U}}(u)(\mathbf{x})=E u\left(\mathcal{P}^{-1}(\mathbf{x})\right)-u^{L}\left(\mathcal{P}^{-1}(\mathbf{x})\right),
$$


we obtain by (4.2) that

$$
\begin{aligned}
\left\|u-\mathcal{P}_{\mathcal{U}}(u)\right\|_{L^{2}\left(\mathbb{S}^{2}\right)} & =\left(\sum_{\tilde{T}_{i j k} \in \tilde{\mathcal{T}}} \int_{\tilde{T}_{i j k}}\left|u(\mathbf{x})-\mathcal{P}_{\mathcal{U}}(u)(\mathbf{x})\right|^{2} d s(\mathbf{x})\right)^{1 / 2} \\
& =\left(\sum_{\tilde{T}_{i j k} \in \tilde{\mathcal{T}}} \int_{\tilde{T}_{i j k}}\left|E u(\mathbf{x})-u^{L}\left(\mathcal{P}^{-1}(\mathbf{x})\right)\right|^{2} d s(\mathbf{x})\right)^{1 / 2} \\
& \leq 2\left(\sum_{T_{i j k} \in \mathcal{T}} \int_{T_{i j k}}\left|\tilde{u}(\mathbf{y})-u^{L}(\mathbf{y})\right|^{2} d s(\mathbf{y})\right)^{1 / 2} \\
& =2\left(\sum_{T_{i j k} \in \mathcal{T}}\left\|\tilde{u}(\mathbf{y})-u^{L}(\mathbf{y})\right\|_{L^{2}\left(T_{i j k}\right)}^{2}\right)^{1 / 2} \\
& \leq \tilde{C}_{1} h^{2}\left(\sum_{T_{i j k} \in \mathcal{T}}\|\tilde{u}\|_{H^{2}\left(T_{i j k}\right)}^{2}\right)^{1 / 2} \\
& \leq C_{1} h^{2}\left(\sum_{\tilde{T}_{i j k} \in \tilde{\mathcal{T}}}\|u\|_{H^{2}\left(\tilde{T}_{i j k}\right)}^{2}\right)^{1 / 2} \\
& =C_{1} h^{2}\|u\|_{H^{2}\left(\mathbb{S}^{2}\right)}
\end{aligned}
$$

The other estimates can be proved in similar manners. We omit the details.

Next, we provide some equivalence between various norms. For convenience, we make the assumption that that all three angles of $T_{i j k}$ are less than $90^{\circ}$ in this section, such a statement generally holds for the triangles in the spherical centroidal Voronoi meshes with sufficient large (no smaller than 42 , for example for the constant density) number of vertices (generators), or equivalently, sufficient small $h$.

Lemma 1. For any $u_{h} \in \mathcal{U}_{\mathcal{W}}$, there exists some constants $C_{1}, C_{2}>0$

$$
\begin{aligned}
C_{1}\left\|u_{h}\right\|_{0, \mathcal{W}} \leq\left\|u_{h}\right\|_{L^{2}\left(\mathbb{S}^{2}\right)} \leq C_{2}\left\|u_{h}\right\|_{0, \mathcal{W}} \\
C_{3}\left\|u_{h}\right\|_{1, \mathcal{W}} \leq\left\|u_{h}\right\|_{H^{1}\left(\mathbb{S}^{2}\right)} \leq C_{4}\left\|u_{h}\right\|_{1, \mathcal{W}}
\end{aligned}
$$

Proof. First, from (4.2), we have

$$
\begin{array}{r}
\frac{1}{2} \sum_{T_{i j k} \in \mathcal{T}} \int_{T_{i j k}}\left(E u_{h}\right)^{2}(\mathbf{y}) d s(\mathbf{y}) \leq\left\|u_{h}\right\|_{L^{2}\left(\mathbb{S}^{2}\right)}^{2}=\sum_{\tilde{T}_{i j k} \in \tilde{\mathcal{T}}} \int_{\tilde{T}_{i j k}} u_{h}^{2}(\mathbf{x}) d s(\mathbf{x}) \\
\leq 2 \sum_{T_{i j k} \in \mathcal{T}} \int_{T_{i j k}}\left(E u_{h}\right)^{2}(\mathbf{y}) d s(\mathbf{y})
\end{array}
$$

Since $u_{h} \in \mathcal{U}_{\mathcal{W}}, E u_{h}$ is linear on $T_{i j k}$. Then

$$
\begin{array}{r}
\int_{T_{i j k}}\left(E u_{h}\right)^{2}(\mathbf{y}) d s(\mathbf{y})=\frac{1}{12}\left[\left(u_{h}^{2}\left(\mathbf{x}_{i}\right)+u_{h}^{2}\left(\mathbf{x}_{j}\right)+u_{h}^{2}\left(\mathbf{x}_{k}\right)\right)\right. \\
\left.+\left(u_{h}\left(\mathbf{x}_{i}\right)+u_{h}\left(\mathbf{x}_{j}\right)+u_{h}\left(\mathbf{x}_{k}\right)\right)^{2}\right] A\left(T_{i j k}\right)
\end{array}
$$

and consequently,

$$
\begin{aligned}
\frac{1}{12}\left[u_{h}^{2}\left(\mathbf{x}_{i}\right)+u_{h}^{2}\left(\mathbf{x}_{j}\right)+\right. & \left.u_{h}^{2}\left(\mathbf{x}_{k}\right)\right] A\left(T_{i j k}\right) \leq \int_{T_{i j k}}\left(E u_{h}\right)^{2}(\mathbf{y}) d s(\mathbf{y}) \\
& \leq \frac{1}{3}\left[\left(u_{h}^{2}\left(\mathbf{x}_{i}\right)+u_{h}^{2}\left(\mathbf{x}_{j}\right)+u_{h}^{2}\left(\mathbf{x}_{k}\right)\right] A\left(T_{i j k}\right)\right.
\end{aligned}
$$

Let

$$
B_{i j k}=A\left(S_{i}\right) u_{h}^{2}\left(\mathbf{x}_{i}\right)+A\left(S_{j}\right) u_{h}^{2}\left(\mathbf{x}_{j}\right)+A\left(S_{k}\right) u_{h}^{2}\left(\mathbf{x}_{k}\right),
$$


clearly, $A\left(S_{i}\right)+A\left(S_{j}\right)+A\left(S_{k}\right)=A\left(T_{i j k}\right)$ (see Fig. 4.1), we have

$$
\sum_{T_{i j k} \in \mathcal{T}} B_{i j k} \leq\left\|u_{h}\right\|_{0, \mathcal{W}}^{2}=\sum_{i=1}^{n} m\left(V_{i}\right) u_{h}^{2}\left(\mathbf{x}_{i}\right) \leq \sum_{T_{i j k} \in \mathcal{T}} 2 B_{i j k}
$$

Since $A\left(T_{i j k}\right) / 4<A\left(S_{m}\right)<A\left(T_{i j k}\right) / 2$ for $m=i, j, k$, with (4.11), (4.12) and (4.13), we have

$$
C_{1}\left\|u_{h}\right\|_{0, \mathcal{W}} \leq\left\|u_{h}\right\|_{L^{2}\left(\mathbb{S}^{2}\right)} \leq C_{2}\left\|u_{h}\right\|_{0, \mathcal{W}}
$$

for some constants $C_{1}, C_{2}>0$.

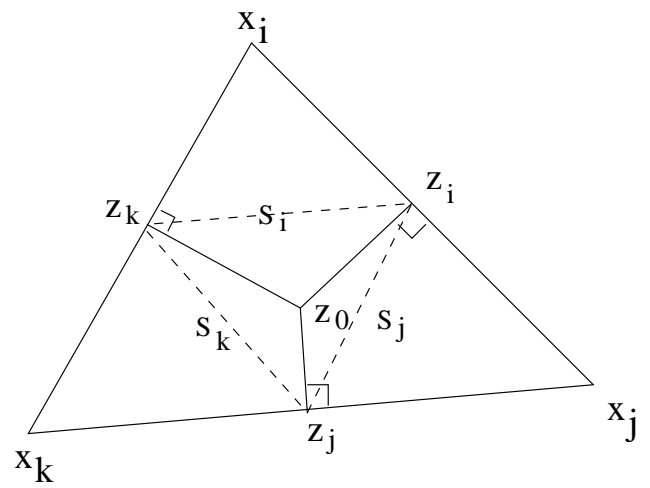

FIG. 4.1. $T_{i j k}$

We now consider $\left|u_{h}\right|_{1, \mathcal{W}}^{2}$ and $\left|u_{h}\right|_{H^{1}\left(\mathbb{S}^{2}\right)}^{2}$. By Proposition 1 and (4.2), we have

$$
\begin{aligned}
\left|u_{h}\right|_{H^{1}\left(\mathbb{S}^{2}\right)}^{2} & =\sum_{\tilde{T}_{i j k} \in \tilde{\mathcal{T}}} \int_{\tilde{T}_{i j k}}\left|\nabla_{s} u_{h}(\mathbf{x})\right|^{2} d s(\mathbf{x}) \\
& =\sum_{\tilde{T}_{i j k} \in \tilde{\mathcal{T}}} \int_{\tilde{T}_{i j k}}\left|\nabla E u_{h}(\mathbf{x})\right|^{2} d s(\mathbf{x}) \\
& \leq 2 \sum_{T_{i j k} \in \mathcal{T}} \int_{T_{i j k}}\left|\nabla E u_{h}(\mathbf{y})\right|^{2} d s(\mathbf{y})
\end{aligned}
$$

and similarly,

$$
\left|u_{h}\right|_{H^{1}\left(\mathbb{S}^{2}\right)}^{2} \geq \frac{1}{2} \sum_{T_{i j k} \in \mathcal{T}} \int_{T_{i j k}}\left|\nabla E u_{h}(\mathbf{y})\right|^{2} d s(\mathbf{y})
$$

Let $\tilde{u}_{h}=\left.E u_{h}\right|_{\mathbf{S}^{*}}$, clearly, for any $\mathbf{y} \in T_{i j k}, \nabla_{*} \tilde{u}_{h}(\mathbf{y})=\vec{g}_{i j k}$ is a constant vector and $g_{i j k} \cdot \overrightarrow{\mathbf{n}}_{T_{i j k}, y}=0$. Since $\overrightarrow{\mathbf{n}}_{\mathbb{S}^{2}, \mathcal{P}(\mathbf{y})} \cdot \overrightarrow{\mathbf{n}}_{T_{i j k}} \geq 1-2 h^{2}$, we have

$$
\left|\nabla_{*} \tilde{u}_{h}(\mathbf{y})\right| \leq\left|\nabla E u_{h}(\mathbf{y})\right| \leq\left(1+2 h^{2}\right)\left|\nabla_{*} \tilde{u}_{h}(\mathbf{y})\right|
$$

which leads to

$$
A\left(T_{i j k}\right)\left|\vec{g}_{i j k}\right|^{2} \leq \int_{T_{i j k}}\left|\nabla E u_{h}(\mathbf{y})\right|^{2} d s(\mathbf{y}) \leq\left(1+2 h^{2}\right)^{2} A\left(T_{i j k}\right)\left|\vec{g}_{i j k}\right|^{2} .
$$


Without loss of generality, suppose that the triangle $\mathcal{T}_{i j k}$ is parallel to the two dimensional $(x, y)$-plane. Again since $E u$ is linear on $\mathcal{T}_{i j k}$, then we have

$$
\begin{gathered}
\nabla_{*} \tilde{u}_{h}=\left(D_{x} E u_{h}, D_{y} E u_{h}, 0\right), \\
\left|g_{i j k}\right|^{2}=\left(D_{x} E u_{h}\right)^{2}+\left(D_{y} E u_{h}\right)^{2}, \\
\left\|\mathbf{x}_{l}-\mathbf{x}_{m}\right\|^{2}=\left(x_{l}-x_{m}\right)^{2}+\left(y_{l}-y_{m}\right)^{2}, \quad l, m=i, j, k,
\end{gathered}
$$

where $\mathbf{x}_{m}=\left(x_{m}, y_{m}, z_{m}\right)$ for $m=i, j, k$. Since $u_{h} \in \mathcal{U}_{\mathcal{W}}$, we have on $\mathcal{T}_{i j k}$,

$$
\left\{\begin{array}{l}
D_{x} E u_{h}=\frac{1}{A\left(T_{i j k}\right)}\left[u_{h}\left(\mathbf{x}_{i}\right)\left(y_{j}-y_{k}\right)+u_{h}\left(\mathbf{x}_{j}\right)\left(y_{k}-y_{i}\right)+u_{h}\left(\mathbf{x}_{k}\right)\left(y_{i}-y_{j}\right)\right], \\
D_{y} E u_{h}=\frac{1}{A\left(T_{i j k}\right)}\left[u_{h}\left(\mathbf{x}_{i}\right)\left(x_{k}-x_{j}\right)+u_{h}\left(\mathbf{x}_{j}\right)\left(x_{i}-x_{k}\right)+u_{h}\left(\mathbf{x}_{k}\right)\left(x_{j}-x_{i}\right)\right] .
\end{array}\right.
$$

So, we finally obtain (see [23]),

$$
\begin{aligned}
\left|u_{h}\right|_{1, \mathcal{W}}^{2}= & \frac{1}{2} \sum_{i=1}^{m} \sum_{j \in \chi_{i}} m\left(\Gamma_{i j}\right) d\left(\mathbf{x}_{i}, \mathbf{x}_{j}\right)\left(\frac{u_{h}\left(\mathbf{x}_{i}\right)-u_{h}\left(\mathbf{x}_{j}\right)}{\left\|\mathbf{x}_{i}-\mathbf{x}_{j}\right\|}\right)^{2} \\
\leq & \left(1+h^{2}\right)^{2} \sum_{T_{i j k} \in \mathcal{T}}\left(2 A\left(\triangle \mathbf{x}_{i} z_{0} \mathbf{x}_{j}\right) G_{i j}+2 A\left(\triangle \mathbf{x}_{j} z_{0} \mathbf{x}_{k}\right) G_{j k}\right. \\
& \left.\quad+2 A\left(\triangle \mathbf{x}_{k} z_{0} \mathbf{x}_{i}\right) G_{k i}\right) \\
\leq & 2 A\left(T_{i j k}\right)\left(\left(D_{x} E u_{h}\left(z_{0}\right)\right)^{2}+\left(D_{y} E u_{h}\left(z_{0}\right)\right)^{2}\right) \\
= & 2 A\left(T_{i j k}\right)\left|\vec{g}_{i j k}\right|^{2}
\end{aligned}
$$

where

$$
\begin{gathered}
A\left(\triangle \mathbf{x}_{l} Q \mathbf{x}_{m}\right)=\frac{1}{2}\left\|\mathbf{x}_{l}-\mathbf{x}_{m}\right\|\left\|z_{l}-z_{0}\right\|, \quad G_{l m}=\left(\frac{u_{h}\left(\mathbf{x}_{l}\right)-u_{h}\left(\mathbf{x}_{m}\right)}{\left\|\mathbf{x}_{l}-\mathbf{x}_{m}\right\|}\right)^{2}, \\
A\left(\triangle \mathbf{x}_{i} z_{0} \mathbf{x}_{j}\right)+A\left(\triangle \mathbf{x}_{j} z_{0} \mathbf{x}_{k}\right)+A\left(\triangle \mathbf{x}_{k} z_{0} \mathbf{x}_{i}\right)=A\left(T_{i j k}\right) .
\end{gathered}
$$

Similarly,

$$
\left|u_{h}\right|_{1, \mathcal{W}}^{2} \geq \frac{1}{2} A\left(T_{i j k}\right)\left|\vec{g}_{i j k}\right|^{2}
$$

Combining (4.15) - (4.20), we have

$$
C_{5}\left|u_{h}\right|_{1, \mathcal{W}}^{2} \leq\left|u_{h}\right|_{H^{1}\left(\mathbb{S}^{2}\right)}^{2} \leq C_{6}\left|u_{h}\right|_{1, \mathcal{W}}^{2}
$$

for some constants $C_{5}, C_{6}>0$. From (4.14) and (4.21), we get that there exist some constants $C_{3}, C_{4}>0$

$$
C_{3}\left\|u_{h}\right\|_{1, \mathcal{W}} \leq\left\|u_{h}\right\|_{H^{1}\left(\mathbb{S}^{2}\right)} \leq C_{4}\left\|u_{h}\right\|_{1, \mathcal{W}}
$$

REMARK 2. The results of the lemma can actually be proved for more general VoronoiDelaunay meshes that satisfies the local mesh regular properties. In fact, we need, for each spherical triangle $\tilde{T}_{i j k}$, that the number of spherical Voronoi regions $V_{m}$ having non-empty $V_{m} \cap \tilde{T}_{i j k}$ is bounded by a constant integer, independent of $\tilde{T}_{i j k}$. Furthermore, any spherical Voronoi region $V_{l}$ can be covered by the union of the finite set of $\tilde{T}_{i j k}$. The maximum number 
of spherical triangles needed in such a union should also be bounded by a constant integer independent of $l$. Under those conditions and the mesh regularity conditions, the above equivalence of norms still holds. The assumption that any triangle only has acute angles makes the above derivation much simpler.

Since $\mathcal{V}_{\mathcal{W}}$ is the space of grid functions on $\mathbb{S}^{2}$ with respect to $\mathcal{W}=\left\{\mathbf{x}_{i}, V_{i}\right\}_{i=1}^{n}$, the set of functions $\left\{\psi_{i}\right\}_{i=1}^{n}$ where

$$
\psi_{i}(\mathbf{x})= \begin{cases}1, & \mathbf{x} \in V_{i} \\ 0, & \mathbf{x} \in \mathbb{S}^{2}-V_{i}\end{cases}
$$

gives a set of basis functions for $\mathcal{V}_{\mathcal{W}}$. For any $u \in H^{1}\left(\mathbb{S}^{2}\right)$ and $v^{h} \in \mathcal{V}_{\mathcal{W}}$, define the bilinear functionals $\mathcal{A}^{*}$ and $\mathcal{A}_{\mathcal{W}}$ such that

$$
\begin{aligned}
& \mathcal{A}^{*}\left(u, v^{h}\right)=\sum_{i=1}^{n} v^{h}\left(\mathbf{x}_{i}\right) \mathcal{A}^{*}\left(u, \psi_{i}\right) \\
& \mathcal{A}_{\mathcal{W}}\left(u, v^{h}\right)=\sum_{i=1}^{n} v^{h}\left(\mathbf{x}_{i}\right) \mathcal{A}_{\mathcal{W}}\left(u, \psi_{i}\right)
\end{aligned}
$$

where

$$
\begin{gathered}
\mathcal{A}^{*}\left(u, \psi_{i}\right)=\int_{\partial V_{i}}\left(-\nabla_{s} u(\mathbf{x})+\overrightarrow{\mathbf{v}}(\mathbf{x}) u(\mathbf{x})\right) \cdot \overrightarrow{\mathbf{n}}_{\mathbf{x}, V_{i}} d \gamma(\mathbf{x})+\int_{V_{i}} b(\mathbf{x}) u(\mathbf{x}) d s(\mathbf{x}) \\
\mathcal{A}_{\mathcal{W}}\left(u, \psi_{i}\right)=\sum_{j \in \chi_{i}} \mathcal{F}_{i j}(u)+\int_{\partial V_{i}} \mathcal{P}_{\mathcal{U}}(u)\left(\overrightarrow{\mathbf{v}}(\mathbf{x}) \cdot \overrightarrow{\mathbf{n}}_{\mathbf{x}, V_{i}}\right) d \gamma(\mathbf{x})+\int_{V_{i}} b \mathcal{P}_{\mathcal{U}}(u) d s(\mathbf{x})
\end{gathered}
$$

Comparing $\mathcal{A}_{\mathcal{W}}$ with the finite volume scheme (3.6), we have in fact replaced $V_{i j}$ by $\int_{\partial V_{i}} \mathcal{P}_{\mathcal{U}}(u)(\overrightarrow{\mathbf{v}}(\mathbf{x})$. $\left.\overrightarrow{\mathbf{n}}_{\mathbf{x}, V_{i}}\right) d \gamma(\mathbf{x})$ and $m\left(V_{i}\right) b_{i} u\left(\mathbf{x}_{i}\right)$ by $\int_{V_{i}} b \mathcal{P}_{\mathcal{U}}(u) d s(\mathbf{x})$. Note that no change is made for a pure diffusion problem containing only second order terms $\nabla_{s} \cdot\left(a(\mathbf{x}) \nabla_{s} u(\mathbf{x})\right)$. Our discrete problem here is then: find $u_{h} \in \mathcal{U}_{\mathcal{W}}$ such that

$$
\mathcal{A}_{\mathcal{W}}\left(u_{h}, v^{h}\right)=\left(f, v^{h}\right), \quad \forall v^{h} \in \mathcal{V}_{\mathcal{W}} .
$$

Formulations like the above for the finite volume methods have been used, for instance, in [23]. The original scheme (3.6) may be viewed as an approximation of (4.24) through numerical integration. It is not difficult to show that the error estimate of Theorem 1 still holds for the above $u_{h}$ using similar analysis in [8]. Combining with Lemma 1 and Proposition 2, we have the following result:

Theorem 2. Suppose that Assumption 1 is satisfied. Let $\mathcal{F}_{i j}$ and $\mathcal{A}_{\mathcal{W}}$ be defined by (3.3) and (4.23) respectively. Then the discrete system (4.24) has a unique solution $u_{h} \in \mathcal{U}_{\mathcal{W}}$. Furthermore, assume that the unique variational solution $u$ of $(1.1)$ belongs to $H^{2}\left(\mathbb{S}^{2}\right)$, then there exists a constant $C>0$ only depending on $\overrightarrow{\mathbf{v}}, b$, and $\sigma$ such that for $e_{h}(\mathbf{x})=u(\mathbf{x})-$ $u_{h}(\mathbf{x})$, we have

$$
\left\|e_{h}\right\|_{H^{1}\left(\mathbb{S}^{2}\right)} \leq C h\|u\|_{H^{2}\left(\mathbb{S}^{2}\right)}
$$


Proof. Notice that $\left\|\mathcal{P}_{\mathcal{U}}(u)-u_{h}\right\|_{1, \mathcal{W}}=\left\|e_{h}\right\|_{1, \mathcal{W}}$, then we have

$$
\begin{aligned}
\left\|e_{h}\right\|_{H^{1}\left(\mathbb{S}^{2}\right)} & =\left\|u-u_{h}\right\|_{H^{1}\left(\mathbb{S}^{2}\right)} \\
& \leq\left\|u-\mathcal{P}_{\mathcal{U}}(u)\right\|_{H^{1}\left(\mathbb{S}^{2}\right)}+\left\|\mathcal{P}_{\mathcal{U}}(u)-u_{h}\right\|_{H^{1}\left(\mathbb{S}^{2}\right)} \\
& \leq C_{1}\|u\|_{H^{2}\left(\mathbb{S}^{2}\right)}+C_{2}\left\|\mathcal{P}_{\mathcal{U}}(u)-u_{h}\right\|_{1, \mathcal{W}} \\
& =C_{1}\|u\|_{H^{2}\left(\mathbb{S}^{2}\right)}+C_{2}\left\|e_{h}\right\|_{1, \mathcal{W}} \\
& \leq C h\|u\|_{H^{2}\left(\mathbb{S}^{2}\right)}
\end{aligned}
$$

where the conclusion of the theorem 1 has been used. $\square$

LEMMA 2. Suppose that $\mathcal{W}=\left\{\mathbf{x}_{i}, V_{i}\right\}_{i=1}^{n}$ is a spherical centroidal Voronoi tessellation of $\mathbb{S}^{2}$ with the density function $\rho$ satisfying $\rho \in C^{1}\left(\mathbb{S}^{2}\right)$ and $\rho(\mathbf{x})>0$ for any $\mathbf{x} \in \mathbb{S}^{2}$. Then, for any $w \in H^{2}\left(\mathbb{S}^{2}\right)$, there exits a constant $C>0$ such that

$$
\left|\int_{V_{i}}\left(w-\mathcal{P}_{\mathcal{V}}(w)\right) d s(\mathbf{x})\right| \leq C h^{2}\left(m\left(V_{i}\right)\right)^{1 / 2}\|w\|_{H^{2}\left(V_{i}\right)}, \quad i=1, \ldots, n
$$

Proof. Let us assume that $w \in C^{2}\left(\mathbb{S}^{2}\right)$, then it is easy to see that $E w \in C^{2}(\Omega)$. Consider the spherical Voronoi region $V_{i}$ associated with $\mathbf{x}_{i}$, for any $\mathbf{x} \in V_{i}$, we have

$$
\begin{array}{r}
w\left(\mathbf{x}_{i}\right)-w(\mathbf{x})=E w\left(\mathbf{x}_{i}\right)-E w(\mathbf{x})=\nabla E w(\mathbf{x}) \cdot\left(\mathbf{x}_{i}-\mathbf{x}\right) \\
+\int_{0}^{1} H(E w)\left(t \mathbf{x}+(1-t) \mathbf{x}_{i}\right)\left(\mathbf{x}_{i}-\mathbf{x}\right) \cdot\left(\mathbf{x}_{i}-\mathbf{x}\right) t d t
\end{array}
$$

where $H(E w)(\mathbf{x})$ denotes the Hessian matrix of $E w$ at $\mathbf{x}$. So

$$
\left|\int_{V_{i}} w-\mathcal{P}_{\mathcal{V}}(w) d s(\mathbf{x})\right| \leq E_{1}+E_{2}+E_{3}
$$

where

$$
\begin{aligned}
& E_{1}=\left|\int_{V_{i}} \nabla E w(\mathbf{x}) \cdot\left(\mathbf{x}_{i}^{*}-\mathbf{x}\right) d s(\mathbf{x})\right|=\left|\int_{V_{i}} \nabla_{s} w(\mathbf{x}) \cdot\left(\mathbf{x}_{i}^{*}-\mathbf{x}\right) d s(\mathbf{x})\right| \\
& E_{2}=\left|\int_{V_{i}} \nabla E w(\mathbf{x}) \cdot\left(\mathbf{x}_{i}-\mathbf{x}_{i}^{*}\right) d s(\mathbf{x})\right|=\left|\int_{V_{i}} \nabla_{s} w(\mathbf{x}) \cdot\left(\mathbf{x}_{i}-\mathbf{x}_{i}^{*}\right) d s(\mathbf{x})\right|, \\
& E_{3}=\int_{V_{i}} \int_{0}^{1}\left|H(E w)\left(t \mathbf{x}+(1-t) \mathbf{x}_{i}\right)\left(\mathbf{x}_{i}-\mathbf{x}\right) \cdot\left(\mathbf{x}_{i}-\mathbf{x}\right)\right| t d t d s(\mathbf{x}) .
\end{aligned}
$$

where $\mathbf{x}_{i}^{*}$ is the standard mass centroid of $V_{i}$ associated with the density function $\rho$.

Using the properties (4.1) of the SCVT, we have

$$
\begin{aligned}
E_{1}= & \left|\int_{V_{i}} \nabla_{s} w(\mathbf{x}) \cdot\left(\mathbf{x}_{i}^{*}-\mathbf{x}\right)-\frac{\rho(\mathbf{x})}{\rho\left(\mathbf{x}_{i}\right)} \Pi_{\mathcal{V}}\left(\nabla_{s} w\right) \cdot\left(\mathbf{x}_{i}^{*}-\mathbf{x}\right) d s(\mathbf{x})\right| \\
\leq & \left|\int_{V_{i}} \frac{\rho(\mathbf{x})-\rho\left(\mathbf{x}_{i}\right)}{\rho\left(\mathbf{x}_{i}\right)} \nabla_{s} w(\mathbf{x}) \cdot\left(\mathbf{x}_{i}^{*}-\mathbf{x}\right) d s(\mathbf{x})\right| \\
& +\left|\int_{V_{i}} \frac{\rho(\mathbf{x})}{\rho\left(\mathbf{x}_{i}\right)}\left(\nabla_{s} w(\mathbf{x})-\Pi_{\mathcal{V}}\left(\nabla_{s} w\right)\right) \cdot\left(\mathbf{x}_{i}^{*}-\mathbf{x}\right) d s(\mathbf{x})\right| \\
= & E_{4}+E_{5}
\end{aligned}
$$

where $\Pi_{\mathcal{V}}$ denotes the $L^{2}$ projection on $\mathcal{V}_{\mathcal{W}}$. 
Since $\mathbb{S}^{2}$ is compact, we have

$$
\begin{aligned}
& E_{4} \leq\left|\int_{V_{i}} \frac{\rho(\mathbf{x})-\rho\left(\mathbf{x}_{i}\right)}{\rho\left(\mathbf{x}_{i}\right)} \nabla_{s} w(\mathbf{x}) \cdot\left(\mathbf{x}_{i}^{*}-\mathbf{x}\right) d s(\mathbf{x})\right| \\
& \leq h \int_{V_{i}} \frac{\max _{\mathbf{x} \in \Omega}|\nabla E \rho(\mathbf{x})|}{\left|\rho\left(\mathbf{x}_{i}\right)\right|}\left|\nabla_{s} w(\mathbf{x})\right|\left\|\mathbf{x}_{i}^{*}-\mathbf{x}\right\| d s(\mathbf{x}) \\
& \leq 2 h^{2} \int_{V_{i}} \frac{\max _{\mathbf{x} \in \Omega}\left|\nabla_{s} \rho(\mathbf{x})\right|}{\left|\rho\left(\mathbf{x}_{i}\right)\right|}\left|\nabla_{s} w(\mathbf{x})\right| d s(\mathbf{x}) \\
& \leq C h^{2} \int_{V_{i}}\left|\nabla_{s} w(\mathbf{x})\right| d s(\mathbf{x}) \\
& \leq C h^{2}\left(\int_{V_{i}}\left|\nabla_{s} w(\mathbf{x})\right|^{2} d s(\mathbf{x})\right)^{1 / 2}\left(\int_{V_{i}} d s(\mathbf{x})\right)^{1 / 2} \\
& \leq C h^{2}\left(m\left(V_{i}\right)\right)^{1 / 2}\|w\|_{H^{2}\left(V_{i}\right)}
\end{aligned}
$$

and

$$
\begin{aligned}
E_{5} & \leq \int_{V_{i}} \frac{\max _{\mathbf{x} \in \Omega}|\rho(\mathbf{x})|}{\left|\rho\left(\mathbf{x}_{i}\right)\right|}\left|\nabla_{s} w(\mathbf{x})-\Pi_{\mathcal{V}}\left(\nabla_{s} w\right)\right|\left\|\mathbf{x}_{i}^{*}-\mathbf{x}\right\| d s(\mathbf{x}) \mid \\
& \leq C\left\|\nabla_{s} w-\Pi_{\mathcal{V}}\left(\nabla_{s} w\right)\right\|_{L^{2}\left(V_{i}\right)}\left(\int_{V_{i}}\left\|\mathbf{x}_{i}^{*}-\mathbf{x}\right\|^{2} d s(\mathbf{x})\right)^{1 / 2} \\
& \leq C h^{2}\left(m\left(V_{i}\right)\right)^{1 / 2}\|w\|_{H^{2}\left(V_{i}\right)}
\end{aligned}
$$

Combining (4.27) and (4.28), we get

$$
E_{1} \leq C h^{2}\left(m\left(V_{i}\right)\right)^{1 / 2}\|w\|_{H^{2}\left(V_{i}\right)} .
$$

Consider $E_{2}$, using the fact that $\mathbf{x}_{i}=\mathbf{x}_{i}^{*} /\left\|\mathbf{x}_{i}^{*}\right\|$ and (4.2), we know that

$$
\left\|x_{i}-\mathbf{x}_{i}^{*}\right\|<h^{2} / 2
$$

Then, we get

$$
\begin{aligned}
E_{2} & \leq h^{2}\left(\int_{V_{i}}\left|\nabla_{s} w(\mathbf{x})\right|^{2} d s(\mathbf{x})\right)^{1 / 2}\left(\int_{V_{i}} d s(\mathbf{x})\right)^{1 / 2} \\
& \leq h^{2}\left(m\left(V_{i}\right)\right)^{1 / 2}\|w\|_{H^{1}\left(V_{i}\right)} .
\end{aligned}
$$

On the other hand, for $E_{3}$, by doing a change of variables $\mathbf{y}=t \mathbf{x}+(1-t) \mathbf{x}_{i}$ and setting $V_{i}^{t}=\left\{\mathbf{y}=t \mathbf{x}+(1-t) \mathbf{x}_{i} \mid \mathbf{x} \in V_{i}\right\}$, we have that $d s(\mathbf{x}) \leq 2 d s(\mathbf{y}) / t^{2}$. Then

$$
E_{3} \leq 2 h^{2} \int_{0}^{1} \int_{V_{i}^{t}} \frac{|H(E w)|}{t} d s(\mathbf{y}) d t
$$

It is easy to see that $m\left(V_{i}^{t}\right) \leq t^{2} m\left(V_{i}\right)$. By a proof similar to that given in [8], we get

$$
\begin{aligned}
E_{3} & \leq 2 h^{2} \int_{0}^{1}\left(\int_{V_{i}^{t}}|H(E w)|^{2} d s(\mathbf{y})\right)^{1 / 2}\left(m\left(V_{i}^{t}\right)\right)^{1 / 2} \frac{1}{t} d t \\
& \leq C h^{2}\left(m\left(V_{i}\right)\right)^{1 / 2}\|w\|_{H^{2}\left(V_{i}\right)} \int_{0}^{1} \frac{1}{t^{2}} d t \\
& \leq C h^{2}\left(m\left(V_{i}\right)\right)^{1 / 2}\|w\|_{H^{2}\left(V_{i}\right)}
\end{aligned}
$$

Finally, we obtain (4.26) for $u \in H^{2}\left(\mathbb{S}^{2}\right)$ by combining (4.29) and (4.30) with (4.31) and invoking a density argument. $\mathrm{C}$ 
THEOREM 3. Let Assumption 1 be satisfied. Suppose that $\mathcal{W}=\left\{\mathbf{x}_{i}, V_{i}\right\}_{i=1}^{n}$ is a spherical centroidal Voronoi tessellation of $\mathbb{S}^{2}$ with the density function $\rho$ satisfying $\rho \in C^{1}\left(\mathbb{S}^{2}\right)$ and $\rho(\mathbf{x})>0$ for any $\mathbf{x} \in \mathbb{S}^{2}$. Let $\mathcal{F}_{i j}$ and $\mathcal{A}_{\mathcal{W}}$ be defined by (3.3) and (4.23) respectively. Then the discrete system (4.24) has a unique solution $u_{h} \in \mathcal{U}_{\mathcal{W}}$. Furthermore, assume that the unique variational solution $u$ of (1.1) belongs to $H^{3}\left(\mathbb{S}^{2}\right)$, then there exists a constant $C>0$ only depending on $\rho, \overrightarrow{\mathbf{v}}, b$, and $\sigma$ such that

$$
\left\|e_{h}\right\|_{L^{2}\left(\mathbb{S}^{2}\right)} \leq C h^{2}\|u\|_{H^{3}\left(\mathbb{S}^{2}\right)}
$$

where $e_{h}(\mathbf{x})=u(\mathbf{x})-u_{h}(\mathbf{x})$.

Proof. For simplicity, we assume $a(\mathbf{x})=1$. Since $u-u_{h} \in H^{1}\left(\mathbb{S}^{2}\right)$, according to (1.4), we know that there exists a weak solution $w \in H^{2}\left(\mathbb{S}^{2}\right)$ satisfying

$$
\mathcal{A}(w, v)=\left(u-u_{h}, v\right), \quad \forall v \in H^{1}\left(\mathbb{S}^{2}\right)
$$

Put $v=u-u_{h}$ in the above equality, then we get

$$
\left\|u-u_{h}\right\|_{L^{2}\left(\mathbb{S}^{2}\right)}^{2}=\left(u-u_{h}, u-u_{h}\right)=\mathcal{A}\left(w, u-u_{h}\right)
$$

Furthermore, from (1.5), we know,

$$
\|w\|_{H^{2}\left(\mathbb{S}^{2}\right)} \leq C|| u-u_{h} \|_{L^{2}\left(\mathbb{S}^{2}\right)}
$$

for some constant $C>0$.

Denote by $\mathcal{P}_{\mathcal{U}}(w)$ and $\mathcal{P}_{\mathcal{V}}(w)$ the interpolants of $w$ on $\mathcal{U}_{\mathcal{W}}$ and $\mathcal{V}_{\mathcal{W}}$ respectively, then we have

$$
\mathcal{A}^{*}\left(u, \mathcal{P}_{\mathcal{V}}(w)\right)=\left(f, \mathcal{P}_{\mathcal{V}}(w)\right), \quad \mathcal{A}_{\mathcal{W}}\left(u_{h}, \mathcal{P}_{\mathcal{V}}(w)\right)=\left(f, \mathcal{P}_{\mathcal{V}}(w)\right)
$$

Consequently, we get

$$
\begin{aligned}
\left\|u-u_{h}\right\|_{L^{2}\left(\mathbb{S}^{2}\right)}^{2} \leq & \left|\mathcal{A}\left(u-u_{h}, w-\mathcal{P}_{\mathcal{U}}(w)\right)\right| \\
& +\left|\mathcal{A}\left(u-u_{h}, \mathcal{P}_{\mathcal{U}}(w)\right)-\mathcal{A}^{*}\left(u-u_{h}, \mathcal{P}_{\mathcal{V}}(w)\right)\right| \\
& +\left|\mathcal{A}^{*}\left(u_{h}, \mathcal{P}_{\mathcal{V}}(w)\right)-\mathcal{A}_{\mathcal{W}}\left(u_{h}, \mathcal{P}_{\mathcal{V}}(w)\right)\right|
\end{aligned}
$$

According to Theorem 2, Proposition 2 and (4.13), we get

$$
\begin{aligned}
\left|\mathcal{A}\left(u-u_{h}, w-\mathcal{P}_{\mathcal{U}}(w)\right)\right| & \leq C\left\|u-u_{h}\right\|_{H^{1}\left(\mathbb{S}^{2}\right)}\left\|w-\mathcal{P}_{\mathcal{U}}(w)\right\|_{H^{1}\left(\mathbb{S}^{2}\right)} \\
& \leq C h^{2}\|u\|_{H^{2}\left(\mathbb{S}^{2}\right)}\|w\|_{H^{2}\left(\mathbb{S}^{2}\right)} \\
& \leq C h^{2}\|u\|_{H^{2}\left(\mathbb{S}^{2}\right)}\left\|u-u_{h}\right\|_{L^{2}\left(\mathbb{S}^{2}\right)}
\end{aligned}
$$


On the other hand, by Green's formula, we have

$$
\begin{aligned}
& \mathcal{A}\left(u-u_{h}, \mathcal{P}_{\mathcal{U}}(w)\right)=\int_{\mathbb{S}^{2}} \nabla_{s}\left(u-u_{h}\right) \cdot \nabla_{s} \mathcal{P}_{\mathcal{U}}(w) d s(\mathbf{x}) \\
& \quad+\int_{\mathbb{S}^{2}}\left(u-u_{h}\right)\left(\overrightarrow{\mathbf{v}} \cdot \nabla_{s} \mathcal{P}_{\mathcal{U}}(w)\right) d s(\mathbf{x})+\int_{\mathbb{S}^{2}} b\left(u-u_{h}\right) \mathcal{P}_{\mathcal{U}}(w) d s(\mathbf{x}) \\
& =\sum_{\tilde{T}_{i j k} \in \tilde{\mathcal{T}}}\left(\int_{\tilde{T}_{i j k}} \nabla_{s}\left(u-u_{h}\right) \cdot \nabla_{s} \mathcal{P}_{\mathcal{U}}(w)+\left(u-u_{h}\right)\left(\overrightarrow{\mathbf{v}} \cdot \nabla_{s} \mathcal{P}_{\mathcal{U}}(w)\right) d s(\mathbf{x})\right) \\
& \quad+\int_{\mathbb{S}^{2}} b\left(u-u_{h}\right) \mathcal{P}_{\mathcal{U}}(w) d s(\mathbf{x}) \\
& =\sum_{\tilde{T}_{i j k} \in \tilde{\mathcal{T}}}\left(\int_{\tilde{T}_{i j k}}-\triangle_{s}\left(u-u_{h}\right) \mathcal{P}_{\mathcal{U}}(w)+\left(\nabla_{s} \cdot\left(u-u_{h}\right) \overrightarrow{\mathbf{v}}\right) \mathcal{P}_{\mathcal{U}}(w) d s(\mathbf{y})\right. \\
& \left.\quad+\int_{\partial \tilde{T}_{i j k}}\left(\nabla_{s}\left(u-u_{h}\right) \cdot \overrightarrow{\mathbf{n}}_{\mathbf{x}, \tilde{T}_{i j k}}\right) \mathcal{P}_{\mathcal{U}}(w)-\left(u-u_{h}\right)\left(\overrightarrow{\mathbf{v}} \cdot \overrightarrow{\mathbf{n}}_{\mathbf{x}, \tilde{T}_{i j k}}\right) \mathcal{P}_{\mathcal{U}}(w) d \gamma(\mathbf{y})\right) \\
& \quad+\int_{\mathbb{S}^{2}} b\left(u-u_{h}\right) \mathcal{P}_{\mathcal{U}}(w) d s(\mathbf{x})
\end{aligned}
$$

where $\overrightarrow{\mathbf{n}}_{\mathbf{x}, \tilde{T}_{i j k}}$ is one of the tangent vector of $\mathbb{S}^{2}$ which is the unit outer normal vector toward the boundary on $\tilde{T}_{i j k}$. Also, we have

$$
\begin{aligned}
\mathcal{A}^{*}\left(u-u_{h}, \mathcal{P}_{\mathcal{V}}(w)\right)=\sum_{i=1}^{n} \mathcal{P}_{\mathcal{V}}(w)\left(\mathbf{x}_{i}\right) \mathcal{A}^{*}\left(u-u_{h}, \psi_{i}\right) \\
=\sum_{i=1}^{n}\left(\int_{\partial V_{i}}-\left(\nabla_{s}\left(u-u_{h}\right) \cdot \overrightarrow{\mathbf{n}}_{\mathbf{x}, V_{i}}\right) \mathcal{P}_{\mathcal{V}}(w)+\left(u-u_{h}\right)\left(\overrightarrow{\mathbf{v}}_{\cdot} \overrightarrow{\mathbf{n}}_{\mathbf{x}, V_{i}}\right) \mathcal{P}_{\mathcal{V}}(w) d \gamma(\mathbf{x})\right. \\
\left.\quad+\int_{V_{i}} b\left(u-u_{h}\right) \mathcal{P}_{\mathcal{V}}(w) d s(\mathbf{x})\right) \\
=\sum_{\tilde{T}_{i j k} \in \tilde{\mathcal{T}} \mathbf{x}_{l} \in T_{i j k}} \int_{\partial V_{l} \cap \tilde{T}_{i j k}}-\left(\nabla_{s}\left(u-u_{h}\right) \cdot \overrightarrow{\mathbf{n}}_{\mathbf{x}, V_{l}}\right) \mathcal{P}_{\mathcal{V}}(w) \\
\quad+\left(u-u_{h}\right)\left(\overrightarrow{\mathbf{v}} \cdot \overrightarrow{\mathbf{n}}_{\mathbf{x}, V_{l}}\right) \mathcal{P}_{\mathcal{V}}(w) d \gamma(\mathbf{x})+\int_{\mathbb{S}^{2}} b\left(u-u_{h}\right) \mathcal{P}_{\mathcal{V}}(w) d s(\mathbf{x}) \\
=\sum_{\tilde{T}_{i j k} \in \tilde{\mathcal{T}}}\left(\int_{\tilde{T}_{i j k}}-\triangle_{s}\left(u-u_{h}\right) \mathcal{P}_{\mathcal{V}}(w)+\left(\nabla_{s} \cdot\left(u-u_{h}\right) \overrightarrow{\mathbf{v}}\right) \mathcal{P}_{\mathcal{V}}(w) d s(\mathbf{x})\right. \\
\left.\quad+\int_{\partial \tilde{T}_{i j k}}\left(\nabla_{s}\left(u-u_{h}\right) \cdot \overrightarrow{\mathbf{n}}_{\mathbf{x}, \tilde{T}_{i j k}}\right) \mathcal{P}_{\mathcal{V}}(w)-\left(u-u_{h}\right)\left(\overrightarrow{\mathbf{v}} \cdot \overrightarrow{\mathbf{n}}_{\mathbf{x}, \tilde{T}_{i j k}}\right) \mathcal{P}_{\mathcal{V}}(w) d \gamma(\mathbf{x})\right) \\
\quad+\int_{\mathbb{S}^{2}} b\left(u-u_{h}\right) \mathcal{P}_{\mathcal{V}}(w) d s(\mathbf{x})
\end{aligned}
$$

So, we obtain

$$
\mathcal{A}\left(u-u_{h}, \mathcal{P}_{\mathcal{U}}(w)\right)-\mathcal{A}^{*}\left(u-u_{h}, \mathcal{P}_{\mathcal{V}}(w)\right)=E_{1}+E_{2}+E_{3}+E_{4}+E_{5}+E_{6}
$$


where

$$
\begin{aligned}
& E_{1}=-\sum_{\tilde{T}_{i j k} \in \tilde{\mathcal{T}}} \int_{\tilde{T}_{i j k}} \triangle_{s} u\left(\mathcal{P}_{\mathcal{U}}(w)-\mathcal{P}_{\mathcal{V}}(w)\right) d s(\mathbf{x}), \\
& E_{2}=\sum_{\tilde{T}_{i j k} \in \tilde{\mathcal{T}}} \int_{\tilde{T}_{i j k}} \triangle_{s} u_{h}\left(\mathcal{P}_{\mathcal{U}}(w)-\mathcal{P}_{\mathcal{V}}(w)\right) d s(\mathbf{x}), \\
& E_{3}=\sum_{\tilde{T}_{i j k} \in \tilde{\mathcal{T}}} \int_{\partial \tilde{T}_{i j k}}\left(\nabla_{s}\left(u-u_{h}\right) \cdot \overrightarrow{\mathbf{n}}_{\mathbf{x}, \tilde{T}_{i j k}}\right)\left(\mathcal{P}_{\mathcal{V}}(w)-\mathcal{P}_{\mathcal{U}}(w)\right) d \gamma(\mathbf{x}), \\
& E_{4}=\sum_{\tilde{T}_{i j k} \in \tilde{\mathcal{T}}} \int_{\tilde{T}_{i j k}}\left(\nabla_{s} \cdot\left(u-u_{h}\right) \overrightarrow{\mathbf{v}}\right)\left(\mathcal{P}_{\mathcal{U}}(w)-\mathcal{P}_{\mathcal{V}}(w)\right) d s(\mathbf{x}) \\
& E_{5}=\sum_{\tilde{T}_{i j k} \in \tilde{\mathcal{T}}} \int_{\partial \tilde{T}_{i j k}}\left(u-u_{h}\right)\left(\overrightarrow{\mathbf{v}} \cdot \overrightarrow{\mathbf{n}}_{\mathbf{x}, \tilde{T}_{i j k}}\right)\left(\mathcal{P}_{\mathcal{V}}(w)-\mathcal{P}_{\mathcal{U}}(w)\right) d \gamma(\mathbf{x}) \\
& E_{6}=\int_{\mathbb{S}^{2}} b\left(u-u_{h}\right)\left(\mathcal{P}_{\mathcal{U}}(w)-\mathcal{P}_{\mathcal{V}}(w)\right) d s(\mathbf{x})
\end{aligned}
$$

Consider $E_{1}$, we have

$$
\begin{aligned}
& \left|E_{1}\right|=\left|\sum_{\tilde{T}_{i j k} \in \tilde{\mathcal{T}}} \int_{\tilde{T}_{i j k}} \triangle_{s} u\left(\mathcal{P}_{\mathcal{U}}(w)-\mathcal{P}_{\mathcal{V}}(w)\right) d s(\mathbf{x})\right| \\
& =\left|\sum_{\tilde{T}_{i j k} \in \tilde{\mathcal{T}}} \int_{\tilde{T}_{i j k}} \triangle_{s} u\left(\mathcal{P}_{\mathcal{U}}(w)-w+w-\mathcal{P}_{\mathcal{V}}(w)\right) d s(\mathbf{x})\right| \\
& \leq\left|\sum_{\tilde{T}_{i j k} \in \tilde{\mathcal{T}}} \int_{\tilde{T}_{i j k}} \triangle_{s} u\left(\mathcal{P}_{\mathcal{U}}(w)-w\right) d s(\mathbf{x})\right| \\
& \quad+\left|\sum_{\tilde{T}_{i j k} \in \tilde{\mathcal{T}}} \int_{\tilde{T}_{i j k}} \triangle_{s} u\left(\mathcal{P}_{\mathcal{V}}(w)-w\right) d s(\mathbf{x})\right| \\
& \leq\left|\sum_{\tilde{T}_{i j k} \in \tilde{\mathcal{T}}} \int_{\tilde{T}_{i j k}} \triangle_{s} u\left(\mathcal{P}_{\mathcal{U}}(w)-w\right) d s(\mathbf{x})\right| \\
& \quad+\left|\sum_{i=1}^{n} \int_{V_{i}} \Pi_{\mathcal{V}}\left(\triangle_{s} u\right)\left(\mathcal{P}_{\mathcal{V}}(w)-w\right) d s(\mathbf{x})\right| \\
& \quad+\left|\sum_{i=1}^{n} \int_{V_{i}}\left(\triangle_{s} u(\mathbf{x})-\Pi_{\mathcal{V}}\left(\triangle_{s} u\right)\right)\left(\mathcal{P}_{\mathcal{V}}(w)-w\right) d s(\mathbf{x})\right|
\end{aligned}
$$

where $\Pi_{\mathcal{V}}$ denotes the $L^{2}$ projection on $\mathcal{V}_{\mathcal{W}}$. Using Lemma 1 and Cauchy-Schwartz inequality, we get

$$
\begin{aligned}
\left|\sum_{\tilde{T}_{i j k} \in \tilde{\mathcal{T}}} \int_{\tilde{T}_{i j k}} \triangle_{s} u\left(\mathcal{P}_{\mathcal{U}}(w)-w\right) d s(\mathbf{x})\right| & \leq C h^{2}\|u\|_{H^{2}\left(\mathbb{S}^{2}\right)}\|w\|_{H^{2}\left(\mathbb{S}^{2}\right)} \\
& \leq C h^{2}\|u\|_{H^{2}\left(\mathbb{S}^{2}\right)}\left\|u-u_{h}\right\|_{L^{2}\left(\mathbb{S}^{2}\right)} .
\end{aligned}
$$


Using Lemma 2, we have

$$
\begin{aligned}
& \left|\sum_{i=1}^{n} \int_{V_{i}} \Pi_{\mathcal{V}}\left(\triangle_{s} u\right)\left(\mathcal{P}_{\mathcal{V}}(w)-w\right) d s(\mathbf{x})\right| \\
& \leq C h^{2} \sum_{i=1}^{n}\left|\Pi_{\mathcal{V}}\left(\triangle_{s} u\right)\right|_{V_{i}} \mid\left(m\left(V_{i}\right)\right)^{1 / 2}\|w\|_{H^{2}\left(V_{i}\right)} \\
& \quad=C h^{2}\left\|\Pi_{\mathcal{V}}\left(\triangle_{s} u\right)\right\|_{L^{2}\left(\mathbb{S}^{2}\right)}\|w\|_{H^{2}\left(\mathbb{S}^{2}\right)}^{2} \\
& \quad \leq C h^{2}\|u\|_{H^{2}\left(\mathbb{S}^{2}\right)}\left\|u-u_{h}\right\|_{L^{2}\left(\mathbb{S}^{2}\right)}
\end{aligned}
$$

and

$$
\begin{array}{r}
\left|\sum_{i=1}^{n} \int_{V_{i}}\left(\triangle_{s} u(\mathbf{x})-\Pi_{\mathcal{V}}\left(\triangle_{s} u\right)\right)\left(\mathcal{P}_{\mathcal{V}}(w)-w\right) d s(\mathbf{x})\right| \leq C h^{2}\|u\|_{H^{3}\left(\mathbb{S}^{2}\right)}\|w\|_{H^{2}\left(\mathbb{S}^{2}\right)} \\
\leq C h^{2}\|u\|_{H^{3}\left(\mathbb{S}^{2}\right)}\left\|u-u_{h}\right\|_{L^{2}\left(\mathbb{S}^{2}\right)}
\end{array}
$$

So, we obtain

$$
\left|E_{1}\right| \leq C h^{2}\|u\|_{H^{3}\left(\mathbb{S}^{2}\right)}\left\|u-u_{h}\right\|_{L^{2}\left(\mathbb{S}^{2}\right)}
$$

As for $E_{2}$, using Corollary 2 and Lemma 1, we have

$$
\begin{aligned}
\left|E_{2}\right| & \leq \sum_{\tilde{T}_{i j k} \in \tilde{\mathcal{T}}} \int_{\tilde{T}_{i j k}}\left|\triangle_{s} u_{h}\left(\mathcal{P}_{\mathcal{U}}(w)-\mathcal{P}_{\mathcal{V}}(w)\right)\right| d s(\mathbf{x}) \\
& \leq C h \sum_{\tilde{T}_{i j k} \in \tilde{\mathcal{T}}} \int_{\tilde{T}_{i j k}}\left|\nabla_{s} u_{h}\right|\left|\left(\mathcal{P}_{\mathcal{U}}(w)-\mathcal{P}_{\mathcal{V}}(w)\right)\right| d s(\mathbf{x}) \\
& \leq C h^{2}\left\|u_{h}\right\|_{H^{1}\left(\mathbb{S}^{2}\right)}\|w\|_{H^{2}\left(\mathbb{S}^{2}\right)} \\
& \leq C h^{2}\left(\|u\|_{H^{1}\left(\mathbb{S}^{2}\right)}+C\|u\|_{H^{2}\left(\mathbb{S}^{2}\right)}\right)\left\|u-u_{h}\right\|_{L^{2}\left(\mathbb{S}^{2}\right)} \\
& \leq C h^{2}\|u\|_{H^{2}\left(\mathbb{S}^{2}\right)}\left\|u-u_{h}\right\|_{L^{2}\left(\mathbb{S}^{2}\right)}
\end{aligned}
$$

According to the continuity of $\nabla_{s} u$ on each $\partial \tilde{T}_{i j k}$, we have

$$
\sum_{\tilde{T}_{i j k} \in \tilde{\mathcal{T}}} \int_{\partial \tilde{T}_{i j k}}\left(\nabla_{s} u \cdot \overrightarrow{\mathbf{n}}_{\mathbf{x}, \tilde{T}_{i j k}}\right)\left(\mathcal{P}_{\mathcal{V}}(w)-\mathcal{P}_{\mathcal{U}}(w)\right) d \gamma(\mathbf{x})=0
$$

so we get

$$
E_{3}=\sum_{\tilde{T}_{i j k} \in \tilde{\mathcal{T}}} \int_{\partial \tilde{T}_{i j k}}\left(\nabla_{s} u_{h} \cdot \overrightarrow{\mathbf{n}}_{\mathbf{x}, \tilde{T}_{i j k}}\right)\left(\mathcal{P}_{\mathcal{V}}(w)-\mathcal{P}_{\mathcal{U}}(w)\right) d \gamma(\mathbf{x})
$$

Additionally, on each edge $\tilde{L}$ of $\tilde{T}_{i j k}$, by symmetry (see Fig. 4.2), we have

$$
\begin{gathered}
\nabla_{s} u_{h}\left(z_{1}\right) \cdot \overrightarrow{\mathbf{n}}_{z_{1}, \tilde{T}_{i j k}}=\nabla_{s} u_{h}\left(z_{2}\right) \cdot \overrightarrow{\mathbf{n}}_{z_{2}, \tilde{T}_{i j k}} \\
\left(\mathcal{P}_{\mathcal{V}}(w)-\mathcal{P}_{\mathcal{U}}(w)\right)\left(z_{1}\right)=-\left(\mathcal{P}_{\mathcal{V}}(w)-\mathcal{P}_{\mathcal{U}}(w)\right)\left(z_{2}\right)
\end{gathered}
$$

which also implies

$$
\int_{\tilde{L}}\left(\nabla_{s} u_{h} \cdot \overrightarrow{\mathbf{n}}_{\mathbf{x}, \tilde{T}_{i j k}}\right)\left(\mathcal{P}_{\mathcal{V}}(w)-\mathcal{P}_{\mathcal{U}}(w)\right) d \gamma(\mathbf{x})=0
$$




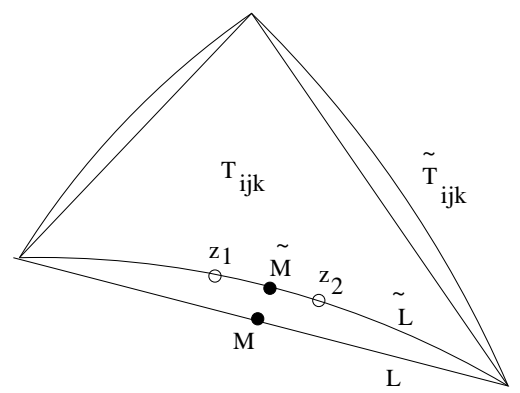

FIG. 4.2. $M, \tilde{M}$ are middle points of $L$ and $\tilde{L}$ respectively, $\left\|z_{1}-\tilde{M}\right\|=\left\|z_{2}-\tilde{M}\right\|$.

So we have

$$
E_{3}=0
$$

About $E_{4}$, we have

$$
\begin{aligned}
\left.\left|E_{4}\right| \leq \sum_{\tilde{T}_{i j k} \in \tilde{\mathcal{T}}} \int_{\tilde{T}_{i j k}} \mid \nabla_{s} \cdot\left(u-u_{h}\right) \overrightarrow{\mathbf{v}}\right)\left(\mathcal{P}_{\mathcal{U}}(w)-\mathcal{P}_{\mathcal{V}}(w) \mid d s(\mathbf{x})\right. \\
\left.\quad \leq \sup _{\mathbf{x} \in \mathbb{S}^{2}}\left(|\overrightarrow{\mathbf{v}}|+\mid \nabla_{s} \overrightarrow{\mathbf{v}}\right) \mid\right) \sum_{\tilde{T}_{i j k} \in \tilde{\mathcal{T}}} \int_{\tilde{T}_{i j k}}\left|\nabla_{s}\left(u-u_{h}\right)\right|\left|\mathcal{P}_{\mathcal{U}}(w)-\mathcal{P}_{\mathcal{V}}(w)\right| d s(\mathbf{x}) \\
\quad \leq C h\left\|u-u_{h}\right\|_{H^{1}\left(\mathbb{S}^{2}\right)}\|w\|_{H^{2}\left(\mathbb{S}^{2}\right)} \\
\quad \leq C h^{2}\|u\|_{H^{2}\left(\mathbb{S}^{2}\right)}\left\|u-u_{h}\right\|_{L^{2}\left(\mathbb{S}^{2}\right)}
\end{aligned}
$$

About $E_{5}$, using Trace theorem [13], we have

$$
\begin{aligned}
\left|E_{5}\right| \leq & \sum_{\tilde{T}_{i j k} \in \tilde{\mathcal{T}}} \int_{\partial \tilde{T}_{i j k}}\left|\left(u-u_{h}\right)\left(\overrightarrow{\mathbf{v}} \cdot \overrightarrow{\mathbf{n}}_{\mathbf{x}, \tilde{T}_{i j k}}\right)\left(\mathcal{P}_{\mathcal{V}}(w)-\mathcal{P}_{\mathcal{U}}(w)\right)\right| d \gamma(\mathbf{x}) \\
\leq & \sup _{\mathbf{x} \in \mathbb{S}^{2}}(|\overrightarrow{\mathbf{v}}|)\left(\sum_{\tilde{T}_{i j k} \in \tilde{\mathcal{T}}} \int_{\partial \tilde{T}_{i j k}}\left|u-u_{h}\right|^{2} d \gamma(\mathbf{x})\right)^{1 / 2} \\
& \cdot\left(\sum_{\tilde{T}_{i j k} \in \tilde{\mathcal{T}}} \int_{\partial \tilde{T}_{i j k}}\left|\mathcal{P}_{\mathcal{V}}(w)-\mathcal{P}_{\mathcal{U}}(w)\right|^{2} d \gamma(\mathbf{x})\right)^{1 / 2} \\
\leq & \left.C \sum_{\tilde{T}_{i j k} \in \tilde{\mathcal{T}}}\left\|u-u_{h}\right\|_{H^{1}\left(\tilde{T}_{i j k}\right)}^{2}\right)^{1 / 2}\left(\sum_{\tilde{T}_{i j k} \in \tilde{\mathcal{T}}} h^{2}\|w\|_{H^{2}\left(\tilde{T}_{i j k}\right)}^{2}\right)^{1 / 2} \\
\leq C h\left\|u-u_{h}\right\|_{H^{1}\left(\mathbb{S}^{2}\right)}\|w\|_{H^{2}\left(\mathbb{S}^{2}\right)} & \leq C h^{2}\|u\|_{H^{2}\left(\mathbb{S}^{2}\right)}\left\|u-u_{h}\right\|_{L^{2}\left(\mathbb{S}^{2}\right)}
\end{aligned}
$$

About $E_{6}$, we have

$$
\begin{aligned}
\left|E_{6}\right| & \leq C\left\|u-u_{h}\right\|_{H^{1}\left(\mathbb{S}^{2}\right)}\left\|\mathcal{P}_{\mathcal{U}}(w)-\mathcal{P}_{\mathcal{V}}(w)\right\|_{L^{2}\left(\mathbb{S}^{2}\right)} \\
& \leq C h^{2}\|u\|_{H^{2}\left(\mathbb{S}^{2}\right)}\|w\|_{H^{1}\left(\mathbb{S}^{2}\right)} \\
& \leq C h^{2}\|u\|_{H^{2}\left(\mathbb{S}^{2}\right)}\left\|u-u_{h}\right\|_{L^{2}\left(\mathbb{S}^{2}\right)}
\end{aligned}
$$

By (4.40), (4.41), (4.43), (4.44) (4.46) and (4.48), we get

$$
\left|\mathcal{A}\left(u-u_{h}, \mathcal{P}_{\mathcal{U}}(w)\right)-\mathcal{A}^{*}\left(u-u_{h}, \mathcal{P}_{\mathcal{V}}(w)\right)\right|=C h^{2}\|u\|_{H^{3}\left(\mathbb{S}^{2}\right)}\left\|u-u_{h}\right\|_{L^{2}\left(\mathbb{S}^{2}\right)}
$$


On the other hand, we have

$$
\begin{aligned}
\mathcal{A}^{*}\left(u_{h}, \mathcal{P}_{\mathcal{V}}(w)\right) & -\mathcal{A}_{\mathcal{W}}\left(u_{h}, \mathcal{P}_{\mathcal{V}}(w)\right)=\sum_{i=1}^{n}\left(\int_{\partial V_{i}}\left(-\nabla_{s} u_{h}(\mathbf{x}) \cdot \overrightarrow{\mathbf{n}}_{\mathbf{x}, V_{i}}\right) \mathcal{P}_{\mathcal{V}}(w) d \gamma(\mathbf{x})\right. \\
& \left.+\int_{\partial V_{i}} u_{h}\left(\overrightarrow{\mathbf{v}} \cdot n_{\mathbf{x}, V_{i}}\right) \mathcal{P}_{\mathcal{V}}(w) d \gamma(\mathbf{x})+\int_{V_{i}} b(\mathbf{x}) u_{h}(\mathbf{x}) \mathcal{P}_{\mathcal{V}}(w) d s(\mathbf{x})\right) \\
& -\sum_{i=1}^{n}\left(\sum_{j \in \chi_{i}} \mathcal{F}_{i j}\left(u_{h}\right) \mathcal{P}_{\mathcal{V}}(w)+\int_{\partial V_{i}} \mathcal{P}_{\mathcal{U}}\left(u_{h}\right)\left(\overrightarrow{\mathbf{v}} \cdot n_{\mathbf{x}, V_{i}}\right) P_{\mathcal{V}}(w) d \gamma(\mathbf{x})\right. \\
& \left.+\int_{V_{i}} b \mathcal{P}_{\mathcal{U}}\left(u_{h}\right) \mathcal{P}_{\mathcal{V}}(w) d s(\mathbf{x})\right) \\
& =E_{7}+E_{8}+E_{9}
\end{aligned}
$$

where

$$
\begin{aligned}
& E_{7}=\sum_{i=1}^{n} \sum_{j \in \chi_{i}} m\left(\Gamma_{i j}\right) \xi_{i j} w\left(x_{i}\right) \\
& E_{8}=\sum_{i=1}^{n} \int_{\partial V_{i}}\left(u_{h}-\mathcal{P}_{\mathcal{U}}\left(u_{h}\right)\right)\left(\overrightarrow{\mathbf{v}} \cdot n_{\mathbf{x}, V_{i}}\right) \mathcal{P}_{\mathcal{V}}(w) d \gamma(\mathbf{x}) \\
& E_{9}=\sum_{i=1}^{n} \int_{V_{i}} b\left(u_{h}-\mathcal{P}_{\mathcal{U}}\left(u_{h}\right)\right) \mathcal{P}_{\mathcal{V}}(w) d s(\mathbf{x})
\end{aligned}
$$

with

$$
\xi_{i j}=-\frac{1}{m\left(\Gamma_{i j}\right)} \int_{\partial \Gamma_{i j}} \nabla_{s} u_{h}(\mathbf{x}) \cdot \overrightarrow{\mathbf{n}}_{\mathbf{x}, V_{i}} d \gamma(\mathbf{x})+\frac{u_{h}\left(\mathbf{x}_{i}\right)-u_{h}\left(\mathbf{x}_{j}\right)}{\left\|\mathbf{x}_{i}-\mathbf{x}_{j}\right\|}
$$

Notice that

$$
\frac{u_{h}\left(\mathbf{x}_{i}\right)-u_{h}\left(\mathbf{x}_{j}\right)}{\left\|\mathbf{x}_{i}-\mathbf{x}_{j}\right\|}=\nabla E u_{h}(\mathbf{y}) \cdot \overrightarrow{\mathbf{n}}_{\mathbf{x}, V_{i}}, \quad \forall \mathbf{x} \in \Gamma_{i j}, \mathbf{y}=\mathcal{P}^{-1}(\mathbf{x})
$$

Since $\|\mathbf{x}-\mathbf{y}\| \leq h^{2} / 2$, we know by Proposition 1 that

$$
\left|\nabla E u_{h}(\mathbf{y})-\nabla_{s} u_{h}(\mathbf{x})\right| \leq 2 h^{2}\left|\nabla E u_{h}(\mathbf{y})\right| .
$$

Then we get

$$
\xi_{i j} \leq 2 h^{2} \frac{\left|u_{h}\left(\mathbf{x}_{i}\right)-u_{h}\left(\mathbf{x}_{j}\right)\right|}{\left\|\mathbf{x}_{i}-\mathbf{x}_{j}\right\|}
$$

It is also easy to find that

$$
E_{7}=\sum_{i=1}^{n} \sum_{j \in \chi_{i}} m\left(\Gamma_{i j}\right) \xi_{i j} w\left(\mathbf{x}_{i}\right)=-\frac{1}{2} \sum_{i=1}^{n} \sum_{j \in \chi_{i}} m\left(\Gamma_{i j}\right) \xi_{i j}\left\|\mathbf{x}_{i}-\mathbf{x}_{j}\right\| \frac{w\left(\mathbf{x}_{i}\right)-w\left(\mathbf{x}_{j}\right)}{\left\|\mathbf{x}_{i}-\mathbf{x}_{j}\right\|}
$$


By the Cauchy-Schwartz inequality and Theorem 2, we have that

$$
\begin{aligned}
\left|E_{7}\right| \leq & \sum_{i=1}^{n} \sum_{j \in \chi_{i}} m\left(\Gamma_{i j}\right) \xi_{i j} d\left(\mathbf{x}_{i}, \mathbf{x}_{j}\right) \frac{\left|w\left(\mathbf{x}_{i}\right)-w\left(\mathbf{x}_{j}\right)\right|}{\left\|\mathbf{x}_{i}-\mathbf{x}_{j}\right\|} \\
\leq & 2\left(\frac{1}{2} \sum_{i=1}^{n} \sum_{j \in \chi_{i}} m\left(\Gamma_{i j}\right) d\left(\mathbf{x}_{i}, \mathbf{x}_{j}\right) \xi_{i j}^{2}\right)^{1 / 2} \\
& \quad \cdot\left(\frac{1}{2} \sum_{i=1}^{n} \sum_{j \in \chi_{i}} m\left(\Gamma_{i j}\right) d\left(\mathbf{x}_{i}, \mathbf{x}_{j}\right)\left(\frac{w\left(\mathbf{x}_{i}\right)-w\left(\mathbf{x}_{j}\right)}{\left\|\mathbf{x}_{i}-\mathbf{x}_{j}\right\|}\right)^{2}\right)^{1 / 2} \\
& \leq 4 h^{2}\left|u_{h}\right|_{1, \mathcal{W}}|w|_{1, \mathcal{W}} \\
\leq & C h^{2}\left\|u_{h}\right\|_{H^{1}\left(\mathbb{S}^{2}\right)}\left|\mathcal{P}_{\mathcal{U}}(w)\right|_{1, \mathcal{W}} \\
\leq & C h^{2}\left\|u_{h}\right\|_{H^{1}\left(\mathbb{S}^{2}\right)}\left|\mathcal{P}_{\mathcal{U}}(w)\right|_{H^{1}\left(\mathbb{S}^{2}\right)} \\
\leq & C h^{2}\left(\|u\|_{H^{1}\left(\mathbb{S}^{2}\right)}+\left\|u-u_{h}\right\|_{H^{1}\left(\mathbb{S}^{2}\right)}\right)\left(\|w\|_{H^{1}\left(\mathbb{S}^{2}\right)}+\left\|w-\mathcal{P}_{\mathcal{U}}(w)\right\|_{H^{1}\left(\mathbb{S}^{2}\right)}\right) \\
\leq & C h^{2}\left(\|u\|_{H^{1}\left(\mathbb{S}^{2}\right)}+C h\|u\|_{H^{2}\left(\mathbb{S}^{2}\right)}\right)\left(\left\|\left.w\right|_{H^{1}\left(\mathbb{S}^{2}\right)}+C h\right\| w \|_{H^{2}\left(\mathbb{S}^{2}\right)}\right) \\
\leq & C h^{2}\|u\|_{H^{2}\left(\mathbb{S}^{2}\right)}\|w\|_{H^{2}\left(\mathbb{S}^{2}\right)} \\
\leq & C h^{2}\|u\|_{H^{2}\left(\mathbb{S}^{2}\right)}\left\|u-u_{h}\right\|_{L^{2}\left(\mathbb{S}^{2}\right)}
\end{aligned}
$$

As for $E_{8}$ and $E_{9}$, since $\mathcal{P}_{\mathcal{U}}\left(u_{h}\right)=u_{h}$, we have

$$
E_{8}=E_{9}=0
$$

So we get

$$
\left|\mathcal{A}_{\mathcal{W}}\left(u_{h}, \mathcal{P}_{\mathcal{V}}(w)\right)-\mathcal{A}^{*}\left(u_{h}, \mathcal{P}_{\mathcal{V}}(w)\right)\right| \leq C h^{2}\|u\|_{H^{2}\left(\mathbb{S}^{2}\right)}\left\|u-u_{h}\right\|_{L^{2}\left(\mathbb{S}^{2}\right)}
$$

By (4.36), (4.49) and (4.54), we get

$$
\left\|u-u_{h}\right\|_{L^{2}\left(\mathbb{S}^{2}\right)}^{2} \leq C h^{2}\|u\|_{H^{3}\left(\mathbb{S}^{2}\right)}\left\|u-u_{h}\right\|_{L^{2}\left(\mathbb{S}^{2}\right)}
$$

which means

$$
\left\|e_{h}\right\|_{L^{2}\left(\mathbb{S}^{2}\right)}=\left\|u-u_{h}\right\|_{L^{2}\left(\mathbb{S}^{2}\right)} \leq C h^{2}\|u\|_{H^{3}\left(\mathbb{S}^{2}\right)} .
$$

This completes the proof of the theorem.

REMARK 3. Extra regularity on the exact solution is required to get the quadratic order error estimates, though in the standard finite element literature, such a requirement is not needed in general. This is seen as a consequence of the quadrature approximations to the standard weak forms of the equations. The regularity in $H^{3}\left(\mathbb{S}^{2}\right)$ can in fact be further weakened to, for instance, $W^{3, p}\left(\mathbb{S}^{2}\right)$ for $p>1$.

REMARK 4. The quadratic order error estimates depend on critically the properties of the spherical centroidal Voronoi meshes. The proof is not valid for a general spherical Voronoi mesh. Such an $L^{2}$ error estimate has not been given in the literature even for the planar finite volume methods based on the general Voronoi-Delaunay meshes. With other choices of the co-volumes which are not of the Voronoi-Delaunay type, a quadratic order estimate has been proved in [23] for two dimensional diffusion equations. A first order $L^{2}$ error estimate has been given in [16]. 
5. Superconvergent gradient recovery. In this section, we discuss how to post-process the finite volume solutions to obtain their tangential gradients in the longitude and latitude directions. Let us rewrite the equation (1.1) in the spherical coordinate system $(\phi, \theta)$ defined by

$$
\mathbf{x}=(r \sin \phi \cos \theta, r \sin \phi \sin \theta, r \cos \phi) \quad \text { for } \quad \phi \in[0, \pi] \quad \text { and } \quad \theta \in[0,2 \pi) .
$$

Ignoring the radial component, we may denote $\overrightarrow{\mathbf{v}}(\phi, \theta)=\left(v_{1}(\phi, \theta), v_{2}(\phi, \theta)\right)$, where $v_{1}$ and $v_{2}$ are the (orthogonal) components of $\overrightarrow{\mathbf{v}}$ in the $\phi$ and $\theta$ directions, respectively, on the tangential surface of $\mathbb{S}^{2}$ at $\mathbf{x}$. We also have

$$
\begin{gathered}
\nabla_{s} u(\phi, \theta)=\left(\frac{1}{r} \frac{\partial u}{\partial \phi}, \frac{1}{r \sin \phi} \frac{\partial u}{\partial \theta}\right) \\
\nabla_{s} \cdot \overrightarrow{\mathbf{v}}(\phi, \theta)=\frac{1}{r \sin \phi}\left(\frac{\partial}{\partial \phi}\left(v_{1} \sin \phi\right)+\frac{\partial v_{2}}{\partial \theta}\right) .
\end{gathered}
$$

For any generator $\mathbf{x}_{i}$ in $\mathcal{W}$, define

$$
V_{\mathbf{x}_{i}}=\cup_{\mathbf{x}_{i} \in T_{i j k}} T_{i j k}
$$

We first project $V_{\mathbf{x}_{i}}$ onto the tangential plane $S_{\mathbf{x}_{i}}$ of $\mathbb{S}^{2}$ at $\mathbf{x}_{i}$, i.e., $S_{\mathbf{x}_{i}}$ is perpendicular to $\overrightarrow{\mathbf{n}}_{\mathbb{S}^{2}, \mathbf{x}_{i}}$ at $\mathbf{x}_{i}$. Denote by $\overrightarrow{\mathbf{n}}_{N, \mathbf{x}_{i}}$ the unit vector on $S_{\mathbf{x}_{i}}$ along the $\phi$ direction. Then, move $S_{\mathbf{x}_{i}}$ to the $(x, y)$-plane by an affine map satisfying that $\overrightarrow{\mathbf{n}}_{\mathbb{S}^{2}, \mathbf{x}_{i}}$ is mapped to the $z$-axis and $\overrightarrow{\mathbf{n}}_{N, \mathbf{x}_{i}}$ to the $x$-axis. See Fig. 5.1. We now define a map $H_{\mathbf{x}_{i}}: V_{i} \rightarrow \mathbb{R}^{2}$ by the above procedure such as $V_{i}^{\prime}=H_{\mathbf{x}_{i}}\left(V_{i}\right)$.

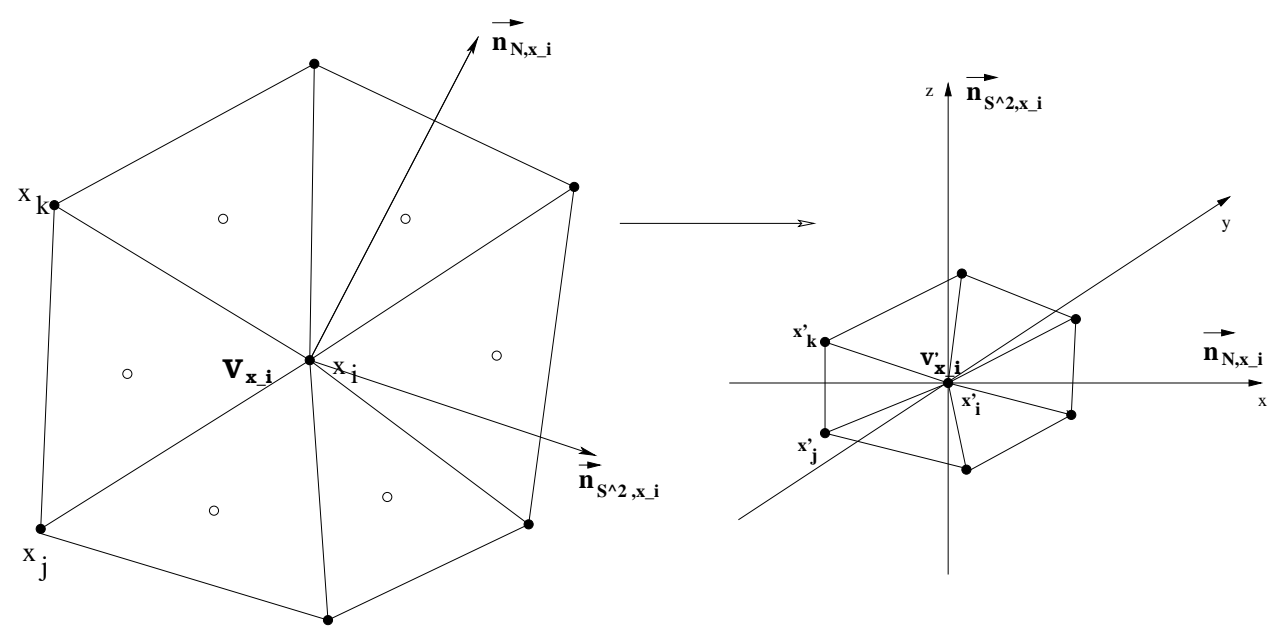

FIG. 5.1. The mapping $H_{\mathbf{x}_{i}}$.

On each planar triangle $\triangle \mathbf{x}_{i}^{\prime} \mathbf{x}_{j}^{\prime} \mathbf{x}_{k}^{\prime}=H_{\mathbf{x}_{i}}\left(T_{i j k}\right)$, we uniquely determine a linear function $\bar{u}_{T_{i j k}}$ by setting

$$
\bar{u}_{T_{i j k}}\left(\mathbf{x}_{i}^{\prime}\right)=u_{h}\left(\mathbf{x}_{i}\right), \quad \bar{u}_{T_{i j k}}\left(\mathbf{x}_{j}^{\prime}\right)=u_{h}\left(\mathbf{x}_{j}\right), \quad \bar{u}_{T_{i j k}}\left(\mathbf{x}_{k}^{\prime}\right)=u_{h}\left(\mathbf{x}_{k}\right) .
$$

Now we define

$$
\bar{\nabla}_{s} u_{h}\left(\mathbf{x}_{i}\right)=\frac{1}{q} \sum_{T_{i j k} \subset V_{\mathbf{x}_{i}}}\left(\frac{\partial \bar{u}_{T_{i j k}}}{\partial x}, \frac{\partial \bar{u}_{T_{i j k}}}{\partial y}\right)
$$


where $q=\operatorname{Card}\left(\left\{T_{i j k} \mid T_{i j k} \subset V_{\mathbf{x}_{i}}\right\}\right)$. We also let

$$
D\left(u, u_{h}\right)=\left(\sum_{i \in I}\left|\nabla_{s} u\left(\mathbf{x}_{i}\right)-\bar{\nabla}_{s} u_{h}\left(\mathbf{x}_{i}\right)\right|^{2} m\left(V_{i}\right)\right)^{1 / 2} .
$$

The index set $I$ may be taken to be the set of all Voronoi generators or a large portion of the generator set. In light of the recent studies on the finite element gradient recovery [34] at mesh symmetric points, the close relationship between finite element and finite volume schemes $[4,32]$, and the nice properties of SCVM, we expect that for the finite volume solution with SCVM, there exists the estimate:

$$
D\left(u, u_{h}\right)=O\left(h^{2}\right) .
$$

Such results are to be numerically investigated in the next section.

6. Numerical experiments. Let $\mathbb{S}^{2}$ be the unit sphere. We now present numerical results that are summarized in the following two examples with each example containing two separate experiments (corresponding to two different exact solutions) but with one identical exact solution. In our experiments, the finite volume meshes are taken to be the spherical centroidal Voronoi meshes corresponding to a constant density function with various different numbers of generators.

For our first example, we choose the exact solution to be

$$
u(\phi, \theta)=\sin ^{2} \phi \cos ^{2} \theta
$$

and study two different model problems whose data are given in Table 6.1.

TABLE 6.1

Data for two model problems.

\begin{tabular}{|c|l||c|c|c|c|}
\hline \multicolumn{2}{|c||}{} & $a(\phi, \theta)$ & $v_{1}(\phi, \theta)$ & $v_{2}(\phi, \theta)$ & $b(\phi, \theta)$ \\
\hline \hline I & no convection & 1 & 0 & 0 & 1 \\
\hline II & convection dominated & 0.05 & $1+\sin \phi$ & $1+\sin \theta$ & $3.0+\sin ^{2} \phi$ \\
\hline
\end{tabular}

Approximate solutions were obtained using the finite volume scheme (3.6) with the central difference scheme and the uniformly distributed spherical centroidal Voronoi meshes in Fig. 2.1 which were based on the constant density function $\rho\left(x_{1}, x_{2}, x_{3}\right)=1$. In Table 6.2, errors in the approximate solution are plotted against the number of generators.

For the second example, the exact solution of (1.1) is chosen to be

$$
u(\phi, \theta))=\sin ^{2}(2 \phi) \cos (4 \theta) .
$$

In Table 6.3, errors in the approximate solution against the number of generators are given. Since the exact solution (6.2) is more complex than (6.1), the largest $2 \%$ of the pointwise gradient errors $\left|\nabla_{s} u\left(\mathbf{x}_{i}\right)-\bar{\nabla}_{s} u_{h}\left(\mathbf{x}_{i}\right)\right|$ was removed from the estimate when computing the $D\left(u, u_{h}\right)$ in Table 6.3. These relatively larger errors concentrates near the 12 defect points of the SCVM (i.e., those Voronoi cells with only 5 neighbors) where the mesh lacks perfect symmetry.

From the numerical values given in the tables we see that, for both the $L^{2}$ errors and the gradient recovery errors $D\left(u, u_{h}\right)$, the trend of quadratic order convergence is very evident as we refine the mesh.

In Fig. 6.1, the errors of the finite volume approximation were plotted (with only half of the sphere shown). The numerical solutions corresponds to those simulations with $n=$ 10242. It can be observed that the errors distribute very evenly on the sphere, thus, the SCVM with a constant density is already adequate. 
TABLE 6.2

Finite volume errors for (6.1) vs. the number of generators $n$.

\begin{tabular}{|c|c||c|c|}
\hline$n$ & & $\left\|u-u_{h}\right\|_{L^{2}\left(\mathbb{S}^{2}\right)}$ & $D\left(u, u_{h}\right)$ \\
\hline \hline 162 & I & $4.038 \mathrm{E}-02$ & $1.331 \mathrm{E}-01$ \\
& II & $6.406 \mathrm{E}-02$ & $1.655 \mathrm{E}-01$ \\
\hline 642 & I & $1.021 \mathrm{E}-02$ & $3.444 \mathrm{E}-02$ \\
& II & $1.612 \mathrm{E}-02$ & $4.214 \mathrm{E}-02$ \\
\hline 2562 & I & $2.556 \mathrm{E}-03$ & $8.788 \mathrm{E}-03$ \\
& II & $3.994 \mathrm{E}-03$ & $1.161 \mathrm{E}-02$ \\
\hline 10242 & I & $6.362 \mathrm{E}-04$ & $2.221 \mathrm{E}-03$ \\
& II & $1.004 \mathrm{E}-03$ & $3.072 \mathrm{E}-03$ \\
\hline 40962 & I & $1.631 \mathrm{E}-04$ & $5.269 \mathrm{E}-04$ \\
& II & $2.375 \mathrm{E}-04$ & $8.132 \mathrm{E}-04$ \\
\hline
\end{tabular}

TABLE 6.3

Finite volume errors for (6.2) vs. the number of generators $n$.

\begin{tabular}{|c|c||c|c|}
\hline$n$ & & $\left\|u-u_{h}\right\|_{L^{2}\left(\mathbb{S}^{2}\right)}$ & $D\left(u, u_{h}\right)$ \\
\hline \hline 162 & I & $4.032 \mathrm{E}-01$ & $4.313 \mathrm{E}-00$ \\
& II & $5.962 \mathrm{E}-01$ & $4.314 \mathrm{E}-00$ \\
\hline 642 & I & $1.370 \mathrm{E}-01$ & $1.178 \mathrm{E}-00$ \\
& II & $1.241 \mathrm{E}-01$ & $1.115 \mathrm{E}-00$ \\
\hline 2562 & I & $2.687 \mathrm{E}-02$ & $3.115 \mathrm{E}-01$ \\
& II & $3.033 \mathrm{E}-02$ & $2.908 \mathrm{E}-01$ \\
\hline 10242 & I & $7.445 \mathrm{E}-03$ & $7.902 \mathrm{E}-02$ \\
& II & $7.577 \mathrm{E}-03$ & $7.346 \mathrm{E}-02$ \\
\hline 40962 & I & $2.080 \mathrm{E}-04$ & $1.745 \mathrm{E}-02$ \\
& II & $2.072 \mathrm{E}-04$ & $1.797 \mathrm{E}-02$ \\
\hline
\end{tabular}

7. Conclusion. High quality spherical grids have many applications. Many strategies have already been studied in atmospherical and geophysical simulations [29] for producing good spherical grids such as those based on the concept of discrete geodesic grids and those based on spherical Voronoi-Delaunay tessellations. For global data analysis purposes, ideas such as the EASE-grid [19] or grids based on Platonic solids have also been explored. Though many of these choices lead to good quality grids, it can be argued that the recently proposed concept of SCVT [7, 8] in general gives grids which are superior to most of existing ones. By providing both theoretical and computational evidences, our study here on a finite volume approximation of linear convection diffusion equations defined on a sphere based on the spherical centroidal Voronoi meshes demonstrated further the optimality of the SCVT grids.

In the future, we will make further studies on the properties on the SCVT grids such as the local energy equipartition and hierarchical SCVT grids for multiresolution analysis. The validity of superconvergent gradient recovery estimate 5.5 will be explored through analytical means. We will also study the application of the SCVT grids to more complex physical problems.

\section{REFERENCES}

[1] F. Aurenhammer, Voronoi diagrams - A survey of a fundamental geometric data structure, ACM Comput. Surv., 23 1991, pp.345-405. 

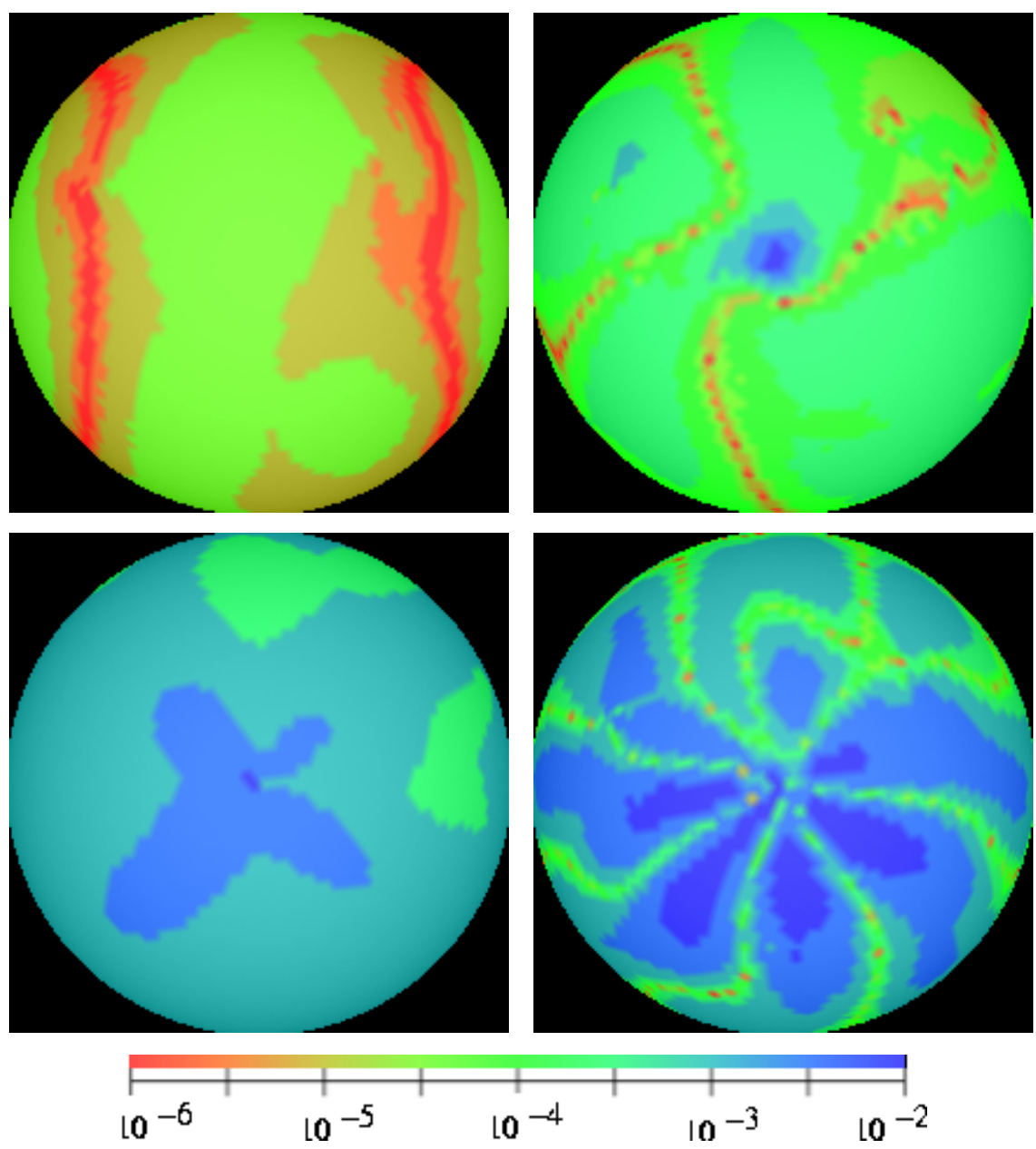

FIG. 6.1. Plots of $\left|u-u_{h}\right|$ on half a sphere. Top row: the example with exact solution (6.1); second row: with exact solution (6.2); left: model I; right: model II; Bottom row: legend.

[2] L. Baughman and N. Walkington, Co-volume methods for degenerate parabolic problems, Numer. Math., 64 1993, pp.45-67.

[3] J. BAUMgaedner AND P. FRederickson, Icosahedral discretization of the two-sphere, SIAM J. Numer. Anal., 22 1985, pp.1107-1115.

[4] Z. CAI, On the finite volume element method, Numer. Math., 58 1991, pp.713-735.

[5] Y. Coudière, T. GallouËT, AND R. Herbin, Discrete Sobolev inequalities and $L^{p}$ error estimates for finite volume solutions of convection diffusion equations, Math. Model. Numer. Anal. 35 2001, pp.767778.

[6] Q. Du, V. Faber and M. Gunzburger, Centroidal Voronoi tessellations: Applications and algorithms, SIAM Review, 41 1999, pp.637-676.

[7] Q. Du, M. Gunzburger, And L. Ju, Constrained centroidal Voronoi tessellations on general surfaces, SIAM J. Sci. Comput., 24 2003, pp. 1488-1506.

[8] Q. Du, M. GunZBurger, AND L. JU, Voronoi-based finite volume methods, optimal Voronoi meshes, and PDEs on the sphere, Comput. Meths. Appl. Mech. Engrg., submitted, 2003;

[9] Q. DU, R.Nicolaides AND X. WU, Analysis and convergence of a covolume approximation of the Ginzburg-Landau model of superconductivity, SIAM J. Numer. Anal., 35 1997, pp. 1049.

[10] G. DZIUK, Finite elements for the Beltrami operator on arbitrary surfaces, Partial Differential Equations and Calculus of Variations, ed. by S. Hildebrandt and R. Leis, Lecture Notes in Mathematics, 1357, Springer, Berlin, 1988, pp.142-155. 
[11] R. Ewing, R. LAZARov, AND P. VAssilevski, Local refinement techniques for elliptic problems on cellcentered grids I. Error analysis, Math. Comp., 56 1991, pp.437-461.

[12] T. Gallouët, R. Herbin, AND M. Vignal, Error estimates on the approximate finite volume solution of convection diffusion equations with general boundary conditions, SIAM J. Numer. Anal., 37 2000, pp.1935-1972.

[13] E. Hebey, Sobolev Spaces on Riemannian Manifolds, Springer, Berlin, 1991.

[14] R. HEIKES AND D. RANDALL, Numerical integration of the shallow-water equations on a twisted icosahedral grid, Monthly Weather Review, 123 1995, pp.1862-1887.

[15] T. Heinze And A. Hense The Shallow Water Equations on the Sphere and their Lagrange-GalerkinSolution, Meteorol. Atmos. Phys., 81 2002, pp.129-137.

[16] R. HERBin, An error estimate for a finite volume scheme for a diffusion-convection problem on a triangular mesh, Num. Meth. PDE, 11 1995, pp.165-173.

[17] J. HeinRich, P. Hyakorn, O. Zienkiewicz, AND A. Mitchell, An "upwind" finite element scheme for wo-dimensional convective transport equations, Int. J. Num. Meth. Engrg. 11 1977, pp.131-143.

[18] L. Ju, Q. Du, AND M. GunZburger, Probabilistic methods for centroidal Voronoi tessellations and their parallel implementations, Paral. Comput., 28 2002, pp.1477-1500.

[19] K. KNOwLES, Points, pixels, grids, and cells - a mapping and gridding primer, Technical Report, National Snow and Ice Data Center, Boulder, CO, 1993.

[20] R. LeVeque And J. Rossmanith, A wave propagation algorithm for the solution of PDEs on the surface of a sphere. International Series of Numerical Mathematics on Hyperbolic Problems, 141 2001, pp.643652.

[21] A. Layton, Cubic Spline Collocation Method for the Shallow Water Equations on the Sphere, Journal of Computational Physics, 179 2002, pp.578-592.

[22] R. LI, Generalized finite difference methods for a nonlinear Dirichlet problem, SIAM J. Numer. Anal, 24 1987, pp.77-88.

[23] R. LI, Z. CHEN, AND W. WU, Generalized difference methods for differential equations, Numerical analysis of finite volume methods, Marcel Dekker, New York, 2000.

[24] R. Lazarov, I. Mishev, AND P. VASSiLeVski, Finite volume methods for convection-diffusion problems, SIAM J. Numer. Anal., 33 1996, pp.31-55.

[25] I. Mishev, Finite volume methods on Voronoi meshes, Num. Meth. PDE, 14 1998, pp.193-212.

[26] K. Morton, Numerical Solution of Convection-Diffusion Problems, Chapman \& Hall, London, 1996.

[27] J. NĔCAS, Les Méthodes Directes en Théorie des Équations Elliptiques, Masson, Paris, 1967.

[28] R. RENKA, ALGORITHM 772 STRIPACK: Delaunay triangulation and Voronoi diagrams on the surface of a sphere, ACM Trans. Math. Soft., 23 1997, pp.416-434.

[29] T. RingleR, Comparing truncation error to Partial Differential Equation solution error on spherical Voronoi Tessellations, Tech report, Department of Atmospheric Science, Colorado State University, 2003.

[30] G. Stuhne AND W. Peltier, New icosahedral grid-point discretizations of the shallow water equations on the sphere, Journal of Computational Physics, 148, 23-58, 1999.

[31] H. Tomita, M. Tsugawa, M.SATOH, AND K.Goto Shallow water model on a modified icosahedral grid by using spring dynamics. Journal of Computational Physics, 174 2001, pp.579-613.

[32] R. VAnselow, Relations between FEM and FVM, in Finite Volumes for Complex Applications: Problems and Perspectives, ed. by F. Benkhaldoun and R. Vilsmerier, Hermes, Paris, 1996.

[33] P. Vassilevski, S. Petrova and R. Lazarov, Finite difference schemes on triangular cell-centered grids with local refinement, SIAM J. Sci. Stat. Comput., 13 1992, pp. 1287-1313.

[34] ZHIMIN ZHANG AND R. LiN, Ultraconvergence of ZZ patch recovery at mesh symmetry points, to appear in Numerische Mathematik. 